\title{
On Minimal Subspaces in Tensor Representations
}

\author{
Antonio Falcó ${ }^{1}$ and Wolfgang Hackbusch ${ }^{2}$ \\ ${ }^{1}$ Universidad CEU Cardenal Herrera \\ San Bartolome 55, 46115 Alfara del Patriarca (Valencia), Spain \\ ${ }^{2}$ Max-Planck-Institut Mathematik in den Naturwissenschaften \\ Inselstr. 22, D-04103 Leipzig, Germany
}

\begin{abstract}
In this paper we introduce and develop the notion of minimal subspaces in the framework of algebraic and topological tensor product spaces. This mathematical structure arises in a natural way in the study of tensor representations. We use minimal subspaces to prove the existence of a best approximation, for any element in a Banach tensor space, by means a tensor given in a typical representation format (Tucker, hierarchical or tensor train). We show that this result holds in a tensor Banach space with a norm stronger that the injective norm and in an intersection of finitely many Banach tensor spaces satisfying some additional conditions. Examples by using topological tensor products of standard Sobolev spaces are given.
\end{abstract}

2010 AMS Subject Classifications: 15A69, 46B28, 46A32

Key words: numerical tensor calculus, tensor product, tensor space, minimal subspaces, weak closedness

\section{Introduction}

Recently, there is an increased interest in numerical methods which make use of tensors. In particular, for high spatial dimensions one must take care that the numerical cost (in time and storage) is linear in the space dimension and does not increase exponentially. Even for three spatial dimensions, these methods can be implied with great success.

A first family of applications using tensor decompositions concerns the extraction of information from complex data. It has been used in many areas such as psychometrics [22, 5], chemometrics [2], analysis of turbulent flows [3], image analysis and pattern recognition [24], data mining.... Another family of applications concerns the compression of complex data (for storage or transmission), also introduced in many areas such as signal processing [15] or computer vision [26]. A survey of tensor decompositions in multilinear algebra and an overview of possible applications can be found in the review paper [14]. In the above applications, the aim is to compress the best as possible the information or to extract a few modes representing some features to be analysed. The use of tensor product approximation is also receiving a growing interest in numerical analysis for the solution of problems defined in high-dimensional tensor spaces, such as PDEs arising in stochastic calculus [1, 4, 9] (e.g., Fokker-Planck equation), stochastic parametric PDEs arising in uncertainty quantification with spectral approaches $[18,8,19]$, and quantum chemistry (cf., e.g., [25]).

Let $d$ vector spaces $V_{j}$ be given (assume, e.g., that $V_{j}=\mathbb{R}^{n_{j}}$ ). The generated tensor space is denoted by $\mathbf{V}={ }_{a} \bigotimes_{j=1}^{d} V_{j}$. A typical representation format is the tensor subspace or Tucker format

$$
\mathbf{u}=\sum_{\mathbf{i} \in \mathbf{I}} \mathbf{a}_{\mathbf{i}} \bigotimes_{j=1}^{d} b_{i_{j}}^{(j)}
$$

where $\mathbf{I}=I_{1} \times \ldots \times I_{d}$ is a multi-index set with $I_{j}=\left\{1, \ldots, r_{j}\right\}, r_{j} \leq \operatorname{dim}\left(V_{j}\right), b_{i_{j}}^{(j)} \in V_{j}\left(i_{j} \in I_{j}\right)$ are (usually orthonormal) basis vectors, and $\mathbf{a}_{\mathbf{i}} \in \mathbb{R}$. Here, $i_{j}$ are the components of $\mathbf{i}=\left(i_{1}, \ldots, i_{d}\right)$. The data size is determined by the numbers $r_{j}$ collected in the tuple $\mathbf{r}:=\left(r_{1}, \ldots, r_{d}\right)$. The set of all tensors representable by (1.1) with fixed $\mathbf{r}$ is

$$
\mathcal{T}_{\mathbf{r}}:=\left\{\mathbf{v} \in \mathbf{V}: \begin{array}{l}
\text { there are subspaces } U_{j} \subset V_{j} \text { such that } \\
\operatorname{dim}\left(U_{j}\right)=r_{j} \text { and } \mathbf{v} \in \mathbf{U}:={ }_{a} \bigotimes_{j=1}^{d} U_{j}
\end{array}\right\}
$$


Here, it is important that the description (1.1) with the vectors $b_{i}^{(j)}$ can be replaced by the generated subspace $U_{j}=\operatorname{span}\left\{b_{i}^{(j)}: i \in I_{j}\right\}$. Note that $\mathcal{T}_{\mathbf{r}}$ is neither a subspace of $\mathbf{V}$ nor a convex set.

The question about minimal subspaces arises naturally from (1.2): Given a tensor $\mathbf{v} \in \mathbf{V}$, what are the subspaces $U_{j} \subset V_{j}$ with minimal dimension $r_{j}$ such that $\mathbf{v} \in \bigotimes_{j=1}^{d} U_{j}$ ? In that case, $\mathbf{v} \in \mathcal{T}_{\mathbf{r}}$ holds.

Another natural question is the approximation of some $\mathbf{v} \in \mathbf{V}$ by $\mathbf{u} \in \mathcal{T}_{\mathbf{r}}$ for a fixed $\mathbf{r}$ : Find $\mathbf{u}_{\text {best }} \in \mathcal{T}_{\mathbf{r}}$ such that $\left\|\mathbf{v}-\mathbf{u}_{\text {best }}\right\|$ equals

$$
\inf \left\{\|\mathbf{v}-\mathbf{u}\|: \mathbf{u} \in \mathcal{T}_{\mathbf{r}}\right\}
$$

for a suitable norm. In the finite dimensional case, compactness arguments show the existence of a bestapproximation. In this paper we discuss this question in the infinite dimensional case (i.e., $\operatorname{dim}\left(V_{j}\right)=\infty$, while still $\left.\operatorname{dim}\left(U_{j}\right)=r_{j}<\infty\right)$.

Here, one should note that tensors have properties which are unexpected compared with matrix theory. For instance, one can define another tensor format ( $r$-term or canonical format) as follows. Fix an integer $r \in \mathbb{N}_{0}$ and set

$$
\mathcal{R}_{r}:=\left\{\mathbf{v}=\sum_{i=1}^{r} \bigotimes_{j=1}^{d} u_{i}^{(j)}: u_{i}^{(j)} \in V_{j} \text { for } 1 \leq i \leq r\right\} .
$$

For $d=2, \mathcal{R}_{r}$ corresponds the matrices of rank $\leq r$. Asking for a solution of inf $\left\{\|\mathbf{v}-\mathbf{u}\|: \mathbf{u} \in \mathcal{R}_{r}\right\}$, one finds examples of $\mathbf{v} \in \mathbf{V}$ even for finite dimensional $\mathbf{V}$, but $d \geq 3$, such that there is no minimiser $\mathbf{u}_{\mathrm{best}} \in \mathcal{R}_{r}$ (cf. [6]).

There are other formats with even better properties than (1.2) (cf. [12], [20]), which are again related to subspaces. In these cases, further subspaces like, e.g., $U_{12} \subset U_{1} \otimes U_{2}$ appear. The representation by the hierarchical format from [12] allows all subspaces with dimension not exceeding a given bound. Also for these formats, the results of this paper apply, e.g., they ensure the existence of best approximations.

The formats from [12] and, in particular, the approach in [20] using a linear tree correspond to the so-called matrix product systems used in quantum chemistry (cf. [25]), provided that the underlying graph structure is a tree. As soon as loops appear, the parameters of the representation cannot be described by dimensions of certain subspaces and the results of this paper do not apply.

In the sequel, we define minimal subspaces $U_{j, \min }(\mathbf{v})$ for algebraic tensors $\mathbf{v} \in{ }_{a} \bigotimes_{j=1}^{d} V_{j}$ (cf. Theorem 3.15 ) as well as for topological tensors $\mathbf{v} \in\|\cdot\| \bigotimes_{j=1}^{d} V_{j}$ (cf. Definition 4.11). In Theorem 4.13 we show that even for weakly convergent sequences $\mathbf{v}_{n} \rightarrow \mathbf{v}$, the dimension of the limiting minimal subspace is bounded by

$$
\operatorname{dim} U_{j, \min }(\mathbf{v}) \leq \liminf _{n \rightarrow \infty} \operatorname{dim} U_{j, \min }\left(\mathbf{v}_{n}\right) \quad \text { for all } 1 \leq j \leq d .
$$

Finally, we discuss the nature of the closed subspace $\|\cdot\| \bigotimes_{j=1}^{d} \overline{U_{j, \min }(\mathbf{v})}\|\cdot\|_{j}$. In the algebraic case, we have by definition that $\mathbf{v} \in{ }_{a} \bigotimes_{j=1}^{d} U_{j, \min }(\mathbf{v})$. This property seems not to be obvious for a general topological tensor $\mathbf{v} \in\|\cdot\| \bigotimes_{j=1}^{d} V_{j}$, but we give sufficient conditions for this property. In particular, it holds for Hilbert tensor spaces.

The paper is organised as follows. The next section shortly recalls the properties of tensors which are necessary for our conclusions. Furthermore, we recall well-known results from functional analysis. In Section 3, we introduce the concept of minimal subspaces of an algebraic tensor and describe a characterisation. Next, in Section 4, minimal subspaces are defined and characterised for Banach tensor spaces. Finally, Section 5 is devoted to the proof of the existence of best approximation tensors in $\mathcal{T}_{\mathbf{r}}$ in a Banach tensor space.

\section{Definitions and results about Banach spaces}

In the following, $X$ is a Banach space with norm $\|\cdot\|$. While $X^{\prime}$ denotes the algebraic dual, $X^{*}$ is the dual space of functionals with bounded dual norm $\|\cdot\|_{X^{*}}$ :

$$
\|\varphi\|_{X^{*}}=\sup \left\{|\varphi(x)|: x \in X \text { with }\|x\|_{X} \leq 1\right\}=\sup \left\{|\varphi(x)| /\|x\|_{X}: 0 \neq x \in X\right\} .
$$

This implies that we recover the $\|\cdot\|_{X}$ norm from the dual norm via

$$
\|x\|_{X}=\max \left\{|\varphi(x)|:\|\varphi\|_{X^{*}}=1\right\}=\max \left\{|\varphi(x)| /\|\varphi\|_{X^{*}}: 0 \neq \varphi \in X^{*}\right\} .
$$


By $\mathcal{L}(X, Y)$ we denote the space of continuous linear mapping from $X$ into $Y$. The corresponding operator norm is written as $\|\cdot\|_{Y \leftarrow X} \cdot \mathcal{L}(X, Y)$ is a subspace of the space $L(X, Y)$ of all linear mappings (without topology).

Remark 2.1 Let $\left\{x_{\nu} \in X: 1 \leq \nu \leq n\right\}$ be linearly independent. Then there are functionals $\varphi_{\nu} \in X^{*}$ such that $\varphi_{\nu}\left(x_{\mu}\right)=\delta_{\nu \mu}$. The functionals $\left(\varphi_{\nu}\right)_{\nu=1}^{n}$ are called dual to $\left(x_{\nu}\right)_{\nu=1}^{n}$.

The following result is known as the Lemma of Auerbach and is proved, e.g., in Meise-Vogt [17, Lemma $10.5]$.

Lemma 2.2 For any $n$-dimensional subspace of a Banach space $X$, there exists a basis $\left\{x_{\nu}: 1 \leq \nu \leq n\right\}$ and a corresponding dual basis $\left\{\varphi_{\nu}: 1 \leq \nu \leq n\right\} \subset X^{*}$ such that $\left\|x_{\nu}\right\|=\left\|\varphi_{\nu}\right\|^{*}=1(1 \leq \nu \leq n)$.

Another statement about an $n$-dimensional subspace of a Banach space is proved, e.g., in DeVore-Lorentz [7, Chapter 9, §7] or Meise-Vogt [17, Proposition 10.6].

Theorem 2.3 Let $Y \subset X$ be a subspace of a Banach space $X$ with $\operatorname{dim}(Y) \leq n$. Then there exists a projection $\Phi \in \mathcal{L}(X, X)$ onto $Y$ such that

$$
\|\Phi\|_{X \leftarrow X} \leq \sqrt{n} .
$$

The bound is sharp for general Banach spaces, but can be improved to $\|\Phi\|_{X \leftarrow X} \leq n^{1 / 2-1 / p}$ for $X=L^{p}$.

We recall that a sequence $x_{n} \in X$ is weakly convergent, if $\lim _{n \rightarrow \infty} \varphi\left(x_{n}\right)$ exists for all $\varphi \in X^{*}$. We say that $\left(x_{n}\right)_{n \in \mathbb{N}}$ converges weakly to $x \in X$, if $\lim \varphi\left(x_{n}\right)=\varphi(x)$ for all $\varphi \in X^{*}$. In this case, we write $x_{n} \rightarrow x$. A well-known result is the following bound.

Lemma 2.4 If $x_{n} \rightarrow x$, then $\|x\| \leq \liminf _{n \rightarrow \infty}\left\|x_{n}\right\|$.

Lemma 2.5 Assume $N \in \mathbb{N}$ and $x_{n}^{(i)} \rightarrow x_{\infty}^{(i)}$ for $1 \leq i \leq N$ with linearly independent $x_{\infty}^{(i)} \in X$. Then there is an $n_{0}$ such that for all $n \geq n_{0}$ the $N$-tuples $\left(x_{n}^{(i)}: 1 \leq i \leq N\right)$ are linearly independent.

Proof. There are functionals $\varphi^{(j)} \in X^{*}(1 \leq j \leq N)$ with $\varphi^{(j)}\left(x_{\infty}^{(i)}\right)=\delta_{i j}$ (cf. Remark 2.1). Set

$$
\Delta_{n}:=\operatorname{det}\left(\left(\varphi^{(j)}\left(x_{n}^{(i)}\right)\right)_{i, j=1}^{N}\right) .
$$

$x_{n}^{(i)} \rightarrow x_{\infty}^{(i)}$ implies $\varphi^{(j)}\left(x_{n}^{(i)}\right) \rightarrow \varphi^{(j)}\left(x_{\infty}^{(i)}\right)$. Continuity of the determinant proves $\Delta_{n} \rightarrow \Delta_{\infty}:=$ $\operatorname{det}\left(\left(\delta_{i j}\right)_{i, j=1}^{N}\right)=1$. Hence, there is an $n_{0}$ such that $\Delta_{n}>0$ for all $n \geq n_{0}$, proving linear independence of $\left\{x_{n}^{(i)}: 1 \leq i \leq N\right\}$.

Lemma 2.6 If $X$ is a reflexive Banach space, any bounded sequence $x_{n} \in X$ has a subsequence $x_{n_{i}}$ converging weakly to some $x \in X$.

Definition 2.7 A subset $M \subset X$ is called weakly closed, if $x_{n} \in M$ and $x_{n} \rightarrow x$ implies $x \in M$.

Note that 'weakly closed' is stronger than 'closed', i.e., $M$ weakly closed $\Rightarrow M$ closed.

Theorem 2.8 Let $(X,\|\cdot\|)$ be a reflexive Banach space with a weakly closed subset $\emptyset \neq M \subset X$. Then the following minimisation problem has a solution: For any $x \in X$ there exists $v \in M$ with

$$
\|x-v\|=\min \{\|x-w\|: w \in M\} .
$$

Proof. Choose any sequence $w_{n} \in M$ with $\left\|x-w_{n}\right\| \searrow \inf \{\|x-w\|: w \in M\}$. Since $\left(w_{n}\right)_{n \in \mathbb{N}}$ is a bounded sequence in $X$, Lemma 2.6 ensures the existence of a subsequence $w_{n_{i}} \rightarrow v \in X . v$ belongs to $M$ because $w_{n_{i}} \in M$ and $M$ is weakly closed. Since also $x-w_{n_{i}} \rightarrow x-v$, Lemma 2.4 shows $\|x-v\| \leq \liminf \left\|x-w_{n_{i}}\right\| \leq$ $\inf \{\|x-w\|: w \in M\}$. 


\section{Minimal subspaces in an algebraic tensor space}

\subsection{Algebraic tensor spaces}

\subsubsection{Definitions and elementary facts}

Concerning the definition of the algebraic tensor space ${ }_{a} \bigotimes_{j=1}^{d} V_{j}$ generated from vector spaces $V_{j}(1 \leq j \leq d)$, we refer to Greub [10]. As underlying field we choose $\mathbb{R}$, but the results hold also for $\mathbb{C}$. The suffix ' $a$ ' in ${ }_{a} \bigotimes_{j=1}^{d} V_{j}$ refers to the 'algebraic' nature. By definition, all elements of

$$
\mathbf{V}:={ }_{a} \bigotimes_{j=1}^{d} V_{j}
$$

are finite linear combinations of elementary tensors $\mathbf{v}=\bigotimes_{j=1}^{d} v_{j}\left(v_{j} \in V_{j}\right)$. In $\S 4$, we shall discuss the Banach tensor space obtained as completion of ${ }_{a} \bigotimes_{j=1}^{d} V_{j}$.

Consider a tensor product $\mathbf{V}={ }_{a} \bigotimes_{j=1}^{d} V_{j}$ of vectors spaces and a fixed tensor $\mathbf{v} \in \mathbf{V}$. Among the subspaces $U_{j} \subset V_{j}$ with

$$
\mathbf{v} \in \mathbf{U}:={ }_{a} \bigotimes_{j=1}^{d} U_{j}
$$

we are looking for the smallest ones. We have to show that a minimal subspace $U_{j}$ exists and that these minimal subspaces can be obtained simultaneously in (3.1) for all $1 \leq j \leq d$. We approach the problem in $\S 3.2 .1$ for the matrix case $d=2$. In $\S 4.1$ we replace the tensor product of vector spaces by a tensor product of Banach spaces. The interesting question is how these minimal subspaces behave as a function of $\mathbf{v}$.

An obvious advantage of the formulation (3.1) is the fact that the $U_{j}$ are of finite dimension even if $\operatorname{dim}\left(V_{j}\right)=\infty$, as stated below.

Lemma 3.1 For $\mathbf{v} \in{ }_{a} \bigotimes_{j=1}^{d} V_{j}$ there are always finite dimensional subspaces $U_{j} \subset V_{j}$ satisfying (3.1).

Proof. By definition of the algebraic tensor space, $\mathbf{v} \in{ }_{a} \bigotimes_{j=1}^{d} V_{j}$ means that there is a finite linear combination

$$
\mathbf{v}=\sum_{\nu=1}^{n} \bigotimes_{j=1}^{d} v_{j}^{(\nu)}
$$

for some $n \in \mathbb{N}_{0}$ and $v_{j}^{(\nu)} \in V_{j}$. Define

$$
U_{j}:=\operatorname{span}\left\{v_{j}^{(\nu)}: 1 \leq \nu \leq n\right\} \quad \text { for } 1 \leq j \leq d .
$$

Then $\mathbf{v} \in \mathbf{U}:={ }_{a} \bigotimes_{j=1}^{d} U_{j}$ proves (3.1) with subspaces of $\operatorname{dimension} \operatorname{dim}\left(U_{j}\right) \leq n$.

The following well-known result is formulated for $d=2$.

Lemma 3.2 For any tensor $\mathbf{v} \in V \otimes_{a} W$ there is an $r \in \mathbb{N}_{0}$ and a representation

$$
\mathbf{v}=\sum_{i=1}^{r} v_{i} \otimes w_{i}
$$

with linearly independent vectors $\left\{v_{i}: 1 \leq i \leq r\right\} \subset V$ and $\left\{w_{i}: 1 \leq i \leq r\right\} \subset W$.

Proof. Take any representation $\mathbf{v}=\sum_{i=1}^{n} v_{i} \otimes w_{i}$. If, e.g., the $\left\{v_{i}: 1 \leq i \leq n\right\}$ are not linearly independent, one $v_{i}$ can be expressed by the others. Without loss of generality assume $v_{n}=\sum_{i=1}^{n-1} \alpha_{i} v_{i}$. Then

$$
v_{n} \otimes w_{n}=\left(\sum_{i=1}^{n-1} \alpha_{i} v_{i}\right) \otimes w_{n}=\sum_{i=1}^{n-1} v_{i} \otimes\left(\alpha_{i} w_{n}\right)
$$


shows that $x$ possesses a representation with only $n-1$ terms:

$$
\mathbf{v}=\left(\sum_{i=1}^{n-1} v_{i} \otimes w_{i}\right)+v_{n} \otimes w_{n}=\sum_{i=1}^{n-1} v_{i} \otimes w_{i}^{\prime} \quad \text { with } \quad w_{i}^{\prime}:=w_{i}+\alpha_{i} w_{n} .
$$

Since each reduction step decreases the number of terms by one, this process terminates, i.e., we obtain a representation with linearly independent $v_{i}$ and $w_{i}$.

In accordance with the usual matrix rank we introduce the following definition.

Definition 3.3 The number $r$ appearing in Lemma 3.2 will be called the rank of the tensor $\mathbf{v}$ and denoted by $\operatorname{rank}(\mathbf{v})$.

The following notation and definitions will be useful. We recall that $L(V, W)$ is the space of linear maps from $V$ into $W$, while $V^{\prime}=L(V, \mathbb{R})$ is the algebraic dual. For metric spaces, $\mathcal{L}(V, W)$ denotes the continuous linear maps, while $V^{*}$ is the topological dual.

Let $\mathcal{I}:=\{1, \ldots, d\}$ be the index set of the 'spatial directions'. In the sequel, the index sets $\mathcal{I} \backslash\{j\}$ will appear. Here, we use the abbreviations

$$
\begin{aligned}
\mathbf{V}_{[j]}:={ }_{a} \bigotimes_{k \neq j} V_{k}, & \text { where } \bigotimes_{k \neq j} \text { means } \bigotimes_{k \in \mathcal{I} \backslash\{j\}}, \\
\mathbf{V}_{[j, k]}:={ }_{a} \bigotimes_{s \neq j, k} V_{s}, & \text { where } \bigotimes_{s \neq j, k} \text { means } \bigotimes_{s \in \mathcal{I} \backslash\{j, k\}} .
\end{aligned}
$$

Similarly, elementary tensors $\bigotimes_{k \neq j} v^{(j)}$ are denoted by $\mathbf{v}_{[j]}$.

For vector spaces $V_{j}$ and $W_{j}$ over $\mathbb{R}$, let linear mappings $A_{j}: V_{j} \rightarrow W_{j}(1 \leq j \leq d)$ be given. Then the definition of the elementary tensor

$$
\mathbf{A}=\bigotimes_{j=1}^{d} A_{j}: \mathbf{V}={ }_{a} \bigotimes_{j=1}^{d} V_{j} \longrightarrow \mathbf{W}={ }_{a} \bigotimes_{j=1}^{d} W_{j}
$$

is given by

$$
\mathbf{A}\left(\bigotimes_{j=1}^{d} v^{(j)}\right):=\bigotimes_{j=1}^{d}\left(A_{j} v^{(j)}\right)
$$

Note that (3.4) extends uniquely to a linear mapping $\mathbf{A}: \mathbf{V} \rightarrow \mathbf{W}$.

Remark 3.4 (a) Let $\mathbf{V}:={ }_{a} \bigotimes_{j=1}^{d} V_{j}$ and $\mathbf{W}:={ }_{a} \bigotimes_{j=1}^{d} W_{j}$. Then the linear combinations of tensor products of linear mappings $\mathbf{A}=\bigotimes_{j=1}^{d} A_{j}$ defined by means of (3.4) form a subspace of $L(\mathbf{V}, \mathbf{W})$ :

$$
\bigotimes_{j=1}^{d} L\left(V_{j}, W_{j}\right) \subset L(\mathbf{V}, \mathbf{W})
$$

(b) The special case of $W_{j}=\mathbb{R}$ for all $j$ (implying $\mathbf{W}=\mathbb{R}$ ) reads as ${ }_{a} \bigotimes_{j=1}^{d} V_{j}^{\prime} \subset \mathbf{V}^{\prime}$.

(c) If $\operatorname{dim}\left(V_{j}\right)<\infty$ and $\operatorname{dim}\left(W_{j}\right)<\infty$ for all $j$, the inclusion ' $\subset$ ' in (a) and (b) may be replaced by ' $=$ '.

Often, mappings $\mathbf{A}=\bigotimes_{j=1}^{d} A_{j}$ will appear, where most of the $A_{k}$ are the identity (and therefore $\left.V_{k}=W_{k}\right)$. If $A_{j} \in L\left(V_{j}, W_{j}\right)$ for one $j$, we use the following notation:

$$
\mathbf{i d}_{[j]} \otimes A_{j}:=\underbrace{i d \otimes \ldots \otimes i d}_{j-1 \text { factors }} \otimes A_{j} \otimes \underbrace{i d \otimes \ldots \otimes i d}_{d-j \text { factors }} \in L\left(\mathbf{V}, \mathbf{V}_{[j]} \otimes_{a} W_{j}\right),
$$

provided that it is obvious what component $j$ is meant. By the multiplication rule $\left(\bigotimes_{j=1}^{d} A_{j}\right) \circ\left(\bigotimes_{j=1}^{d} B_{j}\right)=$ $\bigotimes_{j=1}^{d}\left(A_{j} \circ B_{j}\right)$ and since $i d \circ A_{j}=A_{j} \circ i d$, the following identity holds for $j \neq k$ :

$$
\begin{aligned}
& i d \otimes \ldots \otimes i d \otimes A_{j} \otimes i d \otimes \ldots \otimes i d \otimes A_{k} \otimes i d \otimes \ldots \otimes i d \\
& =\left(\mathbf{i d}_{[j]} \otimes A_{j}\right) \circ\left(\mathbf{i d}_{[k]} \otimes A_{k}\right) \\
& =\left(\mathbf{i d}_{[k]} \otimes A_{k}\right) \circ\left(\mathbf{i d} \mathbf{d}_{[j]} \otimes A_{j}\right)
\end{aligned}
$$


(in the first line we assume $j<k$ ). Note that the meaning of $\mathbf{i d}_{[j]}$ and $\mathbf{i d}_{[k]}$ may differ: in the second line of $(3.5 \mathrm{~b}),\left(\mathbf{i d}_{[k]} \otimes A_{k}\right) \in L\left(\mathbf{V}, \mathbf{V}_{[k]} \otimes_{a} W_{k}\right)$ and $\left(\mathbf{i d}_{[j]} \otimes A_{j}\right) \in L\left(\mathbf{V}_{[k]} \otimes_{a} W_{k}, \mathbf{V}_{[j, k]} \otimes_{a} W_{j} \otimes_{a} W_{k}\right)(\mathrm{cf} .(3.3 \mathrm{~b}))$, whereas in the third one $\left(\mathbf{i d}_{[j]} \otimes A_{j}\right) \in L\left(\mathbf{V}, \mathbf{V}_{[j]} \otimes_{a} W_{j}\right)$ and $\left(\mathbf{i d}_{[k]} \otimes A_{k}\right) \in L\left(\mathbf{V}_{[j]} \otimes_{a} W_{j}, \mathbf{V}_{[j, k]} \otimes_{a} W_{k} \otimes_{a} W_{j}\right)$.

Proceeding inductively with this argument over all indices, we obtain

$$
\mathbf{A}=\bigotimes_{j=1}^{d} A_{j}=\left(\mathbf{i d}_{[1]} \otimes A_{1}\right) \circ \cdots \circ\left(\mathbf{i d}_{[d]} \otimes A_{d}\right) .
$$

If $W_{j}=\mathbb{R}$, i.e., if $A_{j}=\varphi_{j} \in V_{j}^{\prime}$ is a linear form, then id $\otimes \varphi_{j} \in L\left(\mathbf{V}, \mathbf{V}_{[j]}\right)$ is used as symbol for $i d \otimes \ldots \otimes i d \otimes \varphi_{j} \otimes i d \otimes \ldots \otimes i d$ defined by

$$
\left(\mathbf{i d}_{[j]} \otimes \varphi_{j}\right)\left(\bigotimes_{s=1}^{d} v_{s}\right)=\varphi_{j}\left(v_{j}\right) \cdot \bigotimes_{k \neq j} v_{k} .
$$

Thus, if $\varphi=\otimes_{j=1}^{d} \varphi_{j} \in \bigotimes_{j=1}^{d} V_{j}^{\prime}$, we can also write

$$
\boldsymbol{\varphi}=\otimes_{j=1}^{d} \varphi_{j}=\left(\mathbf{i d}_{[1]} \otimes \varphi_{1}\right) \circ \cdots \circ\left(\mathbf{i d}_{[d]} \otimes \varphi_{d}\right) .
$$

Consider again the splitting of $\mathbf{V}={ }_{a} \bigotimes_{j=1}^{d} V_{j}$ into $\mathbf{V}=V_{j} \otimes_{a} \mathbf{V}_{[j]}$ with $\mathbf{V}_{[j]}:={ }_{a} \bigotimes_{k \neq j} V_{k}$. For a linear form $\boldsymbol{\varphi}_{[j]} \in \mathbf{V}_{[j]}^{\prime}$, the notation $i d_{j} \otimes \boldsymbol{\varphi}_{[j]} \in L\left(\mathbf{V}, V_{j}\right)$ is used for the mapping

$$
\left(i d_{j} \otimes \boldsymbol{\varphi}_{[j]}\right)\left(\bigotimes_{k=1}^{d} v^{(k)}\right)=\boldsymbol{\varphi}_{[j]}\left(\bigotimes_{k \neq j} v_{k}\right) \cdot v^{(j)} .
$$

If $\varphi_{[j]}=\bigotimes_{k \neq j} \varphi_{k} \in{ }_{a} \bigotimes_{k \neq j} V_{k}^{\prime}$ is an elementary tensor, $\varphi_{[j]}\left(\bigotimes_{k=1}^{d} v^{(k)}\right)=\prod_{k \neq j} \varphi_{j}\left(v_{k}\right)$ holds in (3.5e). Finally, we can write $(3.5 \mathrm{~d})$ as

$$
\varphi=\otimes_{j=1}^{d} \varphi_{j}=\varphi_{j} \circ\left(i d_{j} \otimes \varphi_{[j]}\right)
$$

for $1 \leq j \leq d$.

\subsubsection{Matricisation}

Definition 3.5 For $j \in \mathcal{I}=\{1, \ldots, d\}$, the map $\mathcal{M}_{j}$ is defined as the isomorphism

$$
\begin{aligned}
\mathcal{M}_{j}:{ }_{a} \bigotimes_{k \neq j} V_{k} & \rightarrow V_{j} \otimes_{a} V_{[j]}, \\
\bigotimes_{k \neq j} v_{k} & \mapsto v_{j} \otimes \mathbf{v}_{[j]} \text { with } \mathbf{v}_{[j]}:=\bigotimes_{k \neq j} v_{k} .
\end{aligned}
$$

In the finite dimensional case of $V_{k}=\mathbb{R}^{n_{k}}$, the tensor space $V_{j} \otimes_{a} V_{[j]}$ of order 2 may be considered as a matrix from $\mathbb{R}^{n_{j} \times n_{[j]}}$, where $n_{[j]}=\prod_{k \neq j} n_{k}$. Then, $\mathcal{M}_{j}$ maps a tensor entry $\mathbf{v}_{i_{1}, \ldots, i_{j}, \ldots, i_{d}}$ into the matrix entry $\left(\mathcal{M}_{j}(\mathbf{v})\right)_{i_{j},\left(i_{1}, \ldots, i_{j-1}, i_{j+1}, \ldots, i_{d}\right)}$. As long as we do not consider matrix properties which depend on the ordering of the index set, we need not introduce an ordering of the $(d-1)$-tuple $\left(i_{1}, \ldots, i_{j-1}, i_{j+1}, \ldots, i_{d}\right)$.

Example 3.6 Consider a tensor $\mathbf{v}=\sum_{i=1}^{3} \sum_{j=1}^{2} \sum_{k=1}^{3} a_{i j k} v_{i} \otimes w_{j} \otimes v_{k} \in \mathbb{R}^{3} \otimes \mathbb{R}^{2} \otimes \mathbb{R}^{3}$, where $\left\{v_{1}, v_{2}, v_{3}\right\}$ is a basis of $\mathbb{R}^{3}$ and $\left\{w_{1}, w_{2}\right\}$ a basis of $\mathbb{R}^{2}$. Then $\mathcal{M}_{2}\left(v_{i} \otimes w_{j} \otimes v_{k}\right)=w_{j} \otimes\left(v_{i} \otimes v_{k}\right) \in \mathbb{R}^{2} \otimes \mathbb{R}^{9}$. The lexicographical ordering of $(i, k)$ leads to the matrix

$$
\mathcal{M}_{2}(\mathbf{v})=\left(\begin{array}{lllllllll}
a_{111} & a_{211} & a_{311} & a_{112} & a_{212} & a_{312} & a_{113} & a_{213} & a_{313} \\
a_{121} & a_{221} & a_{321} & a_{122} & a_{222} & a_{322} & a_{123} & a_{223} & a_{323}
\end{array}\right) .
$$

Next, we restrict the considerations to finite dimensional $V_{k}$. Since tensor products of two vectors can be interpreted as matrices, the mapping $\mathcal{M}_{j}$ is named 'matricisation' (or 'unfolding'). The interpretation of tensors $\mathbf{v}$ as matrices enables us to transfer the matrix terminology to $\mathbf{v}$. In particular, we may define the rank of $\mathcal{M}_{j}(\mathbf{v})$ as a property of $\mathbf{v}$. 
Definition 3.7 Let $\operatorname{dim}\left(V_{k}\right)<\infty(k \in \mathcal{I})$. For all $j \in \mathcal{I}$ we define

$$
\operatorname{rank}_{j}(\mathbf{v}):=\operatorname{rank}\left(\mathcal{M}_{j}(\mathbf{v})\right) .
$$

Hitchcock [13, p.170] (1927) introduced $\operatorname{rank}_{j}(\mathbf{v})$ as 'the rank on the $j^{\text {th }}$ index'. For infinite dimensional vector spaces $V_{j}$, the generalisation is given by $\operatorname{rank}_{j}(\mathbf{v}):=\operatorname{dim} U_{j, \min }(\mathbf{v})$, where the minimal subspaces $U_{j, \min }(\mathbf{v})$ will be defined in $\S 3$.

The next result extends Lemma 3.2 .

Lemma 3.8 Assume that $\mathbf{v} \in{ }_{a} \bigotimes_{j=1}^{d} V_{j}$ with $\operatorname{rank}(\mathbf{v})=r$ and $\mathbf{v}=\sum_{\nu=1}^{r} \bigotimes_{j=1}^{d} v_{j}^{(\nu)}$. Then for each $1 \leq j \leq d$, the elementary tensors

$$
\mathbf{v}_{[j]}^{(\nu)}:=\bigotimes_{k \neq j} v_{k}^{(\nu)} \in{ }_{a} \bigotimes_{k \neq j} V_{k} \quad(1 \leq \nu \leq r)
$$

are linearly independent in ${ }_{a} \bigotimes_{k \neq j} V_{k}$.

Proof. Consider, without loss of generality, the case $j=1$. If the tensors $\left\{\mathbf{v}_{[1]}^{(\nu)}: 1 \leq \nu \leq r\right\}$ are linearly dependent, we also may assume, without loss of generality, that $\mathbf{v}_{[1]}^{(r)}$ may be expressed as $\mathbf{v}_{[1]}^{(r)}=\sum_{\nu=1}^{r-1} \beta_{\nu} \mathbf{v}_{[1]}^{(\nu)}$. Then

$$
\mathbf{v}=\sum_{\nu=1}^{r-1} v_{1}^{(\nu)} \otimes \mathbf{v}_{[1]}^{(\nu)}+v_{1}^{(r)} \otimes \mathbf{v}_{[1]}^{(r)}=\sum_{\nu=1}^{r-1}\left(v_{1}^{(\nu)}+\beta_{\nu} v_{1}^{(r)}\right) \otimes \mathbf{v}_{[1]}^{(\nu)}
$$

implies that $\operatorname{rank}(\mathbf{v})<r$ in contradiction to the minimality of $r$.

\subsection{Minimal subspaces}

\subsubsection{Case $d=2$}

The matrix case $d=2$ will serve as start of an induction. To ensure the existence of minimal subspaces $U_{1}, U_{2}$ with $\mathbf{v} \in U_{1} \otimes U_{2}$, we need a lattice structure, which is subject of the next lemma.

Lemma 3.9 Assume that $X_{i}$ and $Y_{i}$ are subspaces of $V_{i}$ for $i=1,2$. Then

$$
\left(X_{1} \otimes_{a} X_{2}\right) \cap\left(Y_{1} \otimes_{a} Y_{2}\right)=\left(X_{1} \cap Y_{1}\right) \otimes_{a}\left(X_{2} \cap Y_{2}\right) .
$$

Proof. It is clear that $\left(X_{1} \cap Y_{1}\right) \otimes_{a}\left(X_{2} \cap Y_{2}\right) \subset\left(X_{1} \otimes_{a} X_{2}\right) \cap\left(Y_{1} \otimes_{a} Y_{2}\right)$. It remains to show that $\mathbf{v} \in X_{1} \otimes_{a} X_{2}$ and $\mathbf{v} \in Y_{1} \otimes_{a} Y_{2}$ imply that $\mathbf{v} \in\left(X_{1} \cap Y_{1}\right) \otimes_{a}\left(X_{2} \cap Y_{2}\right)$. By assumption, $\mathbf{v}$ has the two representations

$$
\mathbf{v}=\sum_{\nu=1}^{n_{x}} x_{1}^{(\nu)} \otimes x_{2}^{(\nu)}=\sum_{\nu=1}^{n_{y}} y_{1}^{(\nu)} \otimes y_{2}^{(\nu)} \quad \text { with } x_{i}^{(\nu)} \in X_{i}, y_{i}^{(\nu)} \in Y_{i} .
$$

Thanks to Lemma 3.2, we may assume that $\left\{x_{1}^{(\nu)}\right\},\left\{x_{2}^{(\nu)}\right\},\left\{y_{1}^{(\nu)}\right\},\left\{y_{2}^{(\nu)}\right\}$ are linearly independent. The dual basis $\xi_{2}^{(\nu)} \in X_{2}$ of $\left\{x_{2}^{(\nu)}\right\}$ satisfies $\xi_{2}^{(\nu)}\left(x_{2}^{(\mu)}\right)=\delta_{\nu \mu}$ (cf. Remark 2.1). Application of $i d_{1} \otimes \xi_{2}^{(\mu)}$ to the first representation yields $\left(i d_{1} \otimes \xi_{2}^{(\mu)}\right)(\mathbf{v})=x_{1}^{(\mu)}$, while the second representation leads to $\sum_{\nu=1}^{n_{y}} \xi_{\mu}^{(2)}\left(y_{2}^{(\nu)}\right) y_{1}^{(\nu)}$. The resulting equation $x_{1}^{(\mu)}=\sum_{\nu=1}^{n_{y}} \xi_{2}^{(\mu)}\left(y_{2}^{(\nu)}\right) y_{1}^{(\nu)}$ shows that $x_{1}^{(\nu)} \in Y_{1}$. Using the dual basis $\xi_{1}^{(\mu)}$ of $\left\{x_{1}^{(\nu)}\right\}$ and applying $\xi_{1}^{(\mu)} \otimes i d_{2}$ to $\mathbf{v}$ proves $x_{2}^{(\nu)} \in Y_{2}$. Hence $x_{i}^{(\nu)} \in X_{i} \cap Y_{i}$ is shown, i.e., $\mathbf{v} \in\left(X_{1} \cap Y_{1}\right) \otimes_{a}\left(X_{2} \cap Y_{2}\right)$.

Definition 3.10 For a tensor $\mathbf{v} \in V_{1} \otimes_{a} V_{2}$, the minimal subspaces are denoted by $U_{1, \min }(\mathbf{v})$ and $U_{2, \min }(\mathbf{v})$ defined by the property that $\mathbf{v} \in U_{1} \otimes_{a} U_{2}$ implies $U_{1, \min }(\mathbf{v}) \subset U_{1}$ and $U_{2, \min }(\mathbf{v}) \subset U_{2}$, while $\mathbf{v} \in U_{1, \min }(\mathbf{v}) \otimes_{a}$ $U_{2, \min }(\mathbf{v})$. 
For each $\mathbf{v}$, we introduce the family $\mathcal{F}(\mathbf{v})$ as set of pairs $\left(U_{1}, U_{2}\right)$ of subspaces with the property $\mathbf{v} \in U_{1} \otimes_{a} U_{2} \subset V_{1} \otimes_{a} V_{2}$. By Lemma 3.9, we can write

$$
\bigcap_{\left(U_{1}, U_{2}\right) \in \mathcal{F}(\mathbf{v})} U_{1} \otimes_{a} U_{2}=\underbrace{\left(\bigcap_{\left(U_{1}, U_{2}\right) \in \mathcal{F}(\mathbf{v})} U_{1}\right)}_{U_{1, \min }(\mathbf{v})} \otimes_{a}^{\left(\bigcap_{U_{2, \min }(\mathbf{v})} U_{2}\right)} .
$$

Hereby, existence and uniqueness of minimal subspaces $U_{j, \min }(\mathbf{v})$ is guaranteed.

Lemma 3.11 Assume that $\mathbf{v}=\sum_{\nu=1}^{r} v_{1}^{(\nu)} \otimes w_{2}^{(\nu)}$ with linearly independent $\left\{v_{1}^{(\nu)}: 1 \leq \nu \leq r\right\}$ and $\left\{w_{2}^{(\nu)}\right.$ : $1 \leq \nu \leq r\}$. Then theses vectors span the minimal spaces:

$$
U_{1, \min }(\mathbf{v})=\operatorname{span}\left\{v_{1}^{(\nu)}: 1 \leq \nu \leq r\right\} \quad \text { and } \quad U_{2, \min }(\mathbf{v})=\operatorname{span}\left\{w_{2}^{(\nu)}: 1 \leq \nu \leq r\right\} .
$$

In particular, both spaces have equal dimension: $\operatorname{dim}\left(U_{1, \min }(\mathbf{v})\right)=\operatorname{dim}\left(U_{2, \min }(\mathbf{v})\right)=r$.

Proof. Apply the proof of Lemma 3.9 to $X_{1}=\operatorname{span}\left\{v_{2}^{(\nu)}: 1 \leq \nu \leq r\right\}, X_{2}=\operatorname{span}\left\{w_{2}^{(\nu)}: 1 \leq \nu \leq r\right\}$ and $Y_{j}=U_{j, \min }(\mathbf{v})$. It shows that $X_{j} \subset U_{j, \min }(\mathbf{v})$. Since a strict inclusion is excluded, $X_{j}=U_{j, \min }(\mathbf{v})$ proves the assertion.

Proposition 3.12 Let $\mathbf{v} \in V_{1} \otimes_{a} V_{2}$. Then the minimal subspaces $U_{1, \min }(\mathbf{v})$ and $U_{2, \min }(\mathbf{v})$ are characterised by

$$
\begin{aligned}
& U_{1, \min }(\mathbf{v})=\left\{\left(i d_{1} \otimes \varphi_{2}\right)(\mathbf{v}): \varphi_{2} \in V_{2}^{\prime}\right\} \\
& U_{2, \min }(\mathbf{v})=\left\{\left(\varphi_{1} \otimes i d_{2}\right)(\mathbf{v}): \varphi_{1} \in V_{1}^{\prime}\right\}
\end{aligned}
$$

Proof. We use the characterisation from Lemma 3.11, $\mathbf{v}=\sum_{\nu=1}^{r} v_{1}^{(\nu)} \otimes w_{2}^{(\nu)}$ with linearly independent $\left\{v_{1}^{(\nu)}: 1 \leq \nu \leq r\right\}$ and $\left\{w_{2}^{(\nu)}: 1 \leq \nu \leq r\right\}$ spanning the minimal subspaces. Then for each $\varphi_{2} \in V_{2}^{\prime}$ we have

$$
\left(i d_{1} \otimes \varphi_{2}\right)(\mathbf{v})=\sum_{\mu=1}^{r} \varphi_{2}\left(w_{2}^{(\mu)}\right) v_{1}^{(\mu)} \in U_{1, \min }(\mathbf{v}) .
$$

From the proof of Lemma 3.9, there are mappings $\left(i d_{1} \otimes \varphi_{2}\right)$ yielding $v_{1}^{(\mu)}$ for any $1 \leq \mu \leq r$; thus,

$$
\left\{\left(i d_{1} \otimes \varphi_{2}\right)(\mathbf{v}): \varphi_{2} \in V_{2}^{\prime}\right\}=U_{1, \min }(\mathbf{v}) .
$$

Analogously, $\left\{\left(\varphi_{1} \otimes i d_{2}\right)(\mathbf{v}): \varphi_{1} \in V_{1}^{\prime}\right\}=U_{2, \min }(\mathbf{v})$ is shown, proving (3.7a) and (3.7b).

For $V_{1}=\mathbb{R}^{n_{1}}$ and $V_{2}=\mathbb{R}^{n_{2}}$, when $V_{1} \otimes_{a} V_{2}$ is isomorphic to matrices from $\mathbb{R}^{n_{1} \times n_{2}}$, definition (3.7a) may be interpreted as $U_{1, \min }(\mathbf{v})=\operatorname{Col} \mathcal{M}_{1}(\mathbf{v})=\operatorname{span}\left\{\mathcal{M}_{1}(\mathbf{v}) x: x \in V_{2}\right\}\left(\mathcal{M}_{1}(\mathbf{v})\right.$ is the matrix corresponding to $\mathbf{v}$, cf. Definition 3.5). Similarly, (3.7b) becomes $U_{1, \min }(\mathbf{v})=\operatorname{Col} \mathcal{M}_{1}(\mathbf{v})^{T}=\operatorname{Col} \mathcal{M}_{2}(\mathbf{v})$.

Corollary 3.13 The following statements hold.

(a) Once $U_{1, \min }(\mathbf{v})$ and $U_{2, \min }(\mathbf{v})$ are given, one may select any basis $\left\{v^{(\nu)}: 1 \leq \nu \leq r\right\}$ of $U_{1, \min }(\mathbf{v})$ (respectively, $\left\{w^{(\nu)}: 1 \leq \nu \leq r\right\}$ of $U_{2, \min }(\mathbf{v})$ ) and finds a representation (3.2) with these $v^{(\nu)}$ (respectively, $\left.w^{(\nu)}\right)$ and some other basis of $U_{2, \min }(\mathbf{v})$ (respectively, $U_{1, \min }(\mathbf{v})$ ). Otherwise, if $\left\{v^{(\nu)}: 1 \leq \nu \leq r\right\}$ is a basis of a subspace $U_{1} \supsetneqq U_{1, \min }(\mathbf{v})$ (respectively, $\left\{w^{(\nu)}: 1 \leq \nu \leq r\right\}$ of $U_{2} \supsetneqq U_{2, \min }(\mathbf{v})$ ), a representation (3.2) still exists, but the $v^{(\nu)}$ (respectively, $\left.w^{(\nu)}\right)$ are linearly dependent.

(b) If we fix a basis $\left\{w^{(\nu)}: 1 \leq \nu \leq r\right\}$ of a subspace $U_{2} \subset V_{2}$, there are mappings $\left\{\varphi^{(\nu)}: 1 \leq \nu \leq r\right\} \subset U_{2}^{\prime}$ such that $\left(i d_{1} \otimes \varphi^{(\nu)}\right)(\mathbf{w}) \in U_{1, \min }(\mathbf{w})$ and

$$
\mathbf{w}=\sum_{\nu=1}^{r}\left(i d_{1} \otimes \varphi^{(\nu)}\right)(\mathbf{w}) \otimes w^{(\nu)} \quad \text { for all } \mathbf{w} \in V_{1} \otimes_{a} U_{2} .
$$


Proof. For statement (a) consider the representation of $\mathbf{v}$ by (3.2) with bases $\left\{v_{i}\right\}_{i=1}^{r}$ and $\left\{w_{i}\right\}_{i=1}^{r}$. Applying a basis transformation $\left\{v_{i}\right\}_{i=1}^{r} \mapsto\left\{\hat{v}_{i}\right\}_{i=1}^{r}$, we obtain $\mathbf{v}=\sum_{i=1}^{r} \hat{v}_{i} \otimes \hat{w}_{i}$ with another basis $\left\{\hat{w}_{i}\right\}_{i=1}^{r}$.

To prove (b) take a basis $\left\{\varphi_{2}^{(\nu)}: 1 \leq \nu \leq r\right\}$ of $U_{2}^{\prime}$ dual to $\left\{w_{2}^{(\nu)}: 1 \leq \nu \leq r\right\}$ and set $\left\{i d_{1} \otimes \varphi_{2}^{(\nu)}: 1 \leq \nu \leq r\right\} \subset L\left(V_{1} \otimes_{a} U_{2}, V_{1}\right)$. By statement (a), any $\mathbf{w} \in V_{1} \otimes U_{2}$ has a representation given by $\mathbf{w}=\sum_{\nu=1}^{r} v_{1}^{(\mu)} \otimes w_{2}^{(\mu)}$, here $\left\{v_{1}^{(\mu)}: 1 \leq \nu \leq r\right\}$ is a basis of $U_{1, \min }(\mathbf{w})$. Then

$$
\left(i d_{1} \otimes \varphi_{2}^{(\nu)}\right)(\mathbf{w})=\sum_{\mu=1}^{r} \varphi_{\nu}\left(w_{2}^{(\mu)}\right) \cdot v_{1}^{(\mu)}=\sum_{\mu=1}^{r} \delta_{\nu, \mu} v_{1}^{(\mu)}=v_{1}^{(\nu)}
$$

holds, proving the assertion.

\subsubsection{Definition in the general case}

In the following, we assume that $d \geq 3$, and generalise some of the features of tensors of second order.

By Lemma 3.1, we may assume $\mathbf{v} \in \mathbf{U}:={ }_{a} \bigotimes_{j=1}^{d} U_{j}$ with finite dimensional subspaces $U_{j} \subset V_{j}$. The lattice structure from Lemma 3.9 generalises to higher order.

Lemma 3.14 Assume that $X_{i}$ and $Y_{i}$ are subspaces of $V_{i}$ for $i=1, \ldots, d$. Then the identity

$$
\left({ }_{a} \bigotimes_{j=1}^{d} X_{j}\right) \cap\left({ }_{a} \bigotimes_{j=1}^{d} Y_{j}\right)={ }_{a} \bigotimes_{j=1}^{d}\left(X_{j} \cap Y_{j}\right)
$$

holds.

Proof. For the start of the induction at $d=2$ use Lemma 3.9. Assume that the assertion holds for $d-1$ and write ${ }_{a} \bigotimes_{j=1}^{d} X_{j}$ as $X_{1} \otimes_{a} X_{[1]}$ with $X_{[1]}:={ }_{a} \bigotimes_{j=2}^{d} X_{j}$. Similarly, use ${ }_{a} \otimes_{j=1}^{d} Y_{j}=Y_{1} \otimes_{a} Y_{[1]}$. Lemma 3.9 states that $\mathbf{v} \in\left(X_{1} \cap Y_{1}\right) \otimes_{a}\left(X_{[1]} \cap Y_{[1]}\right)$. By induction hypothesis, $X_{[1]} \cap Y_{[1]}={ }_{a} \bigotimes_{j=2}^{d}\left(X_{j} \cap Y_{j}\right)$ is valid, proving the assertion.

Again, the minimal subspaces $U_{j, \min }(\mathbf{v})$ are given by the intersection of all $U_{j}$ satisfying $\mathbf{v} \in{ }_{a} \bigotimes_{j=1}^{d} U_{j}$.

The algebraic characterisation of $U_{j, \min }(\mathbf{v})$ is similar as for $d=2$. To this end, we introduce the following two subspaces (recall $(3.5 \mathrm{e}))$ :

$$
\begin{aligned}
U_{j}^{I}(\mathbf{v}) & :=\left\{\left(i d_{k} \otimes \boldsymbol{\varphi}_{[k]}\right)(\mathbf{v}): \boldsymbol{\varphi}_{[j]} \in{ }_{a} \bigotimes_{k \neq j} V_{k}^{\prime}\right\}, \\
U_{j}^{I I}(\mathbf{v}): & =\left\{\left(i d_{j} \otimes \boldsymbol{\varphi}_{[j]}\right)(\mathbf{v}): \boldsymbol{\varphi}_{[j]} \in\left({ }_{a} \bigotimes_{k \neq j} V_{k}\right)^{\prime}\right\} .
\end{aligned}
$$

In the case of a normed space $V_{j}$, we may consider the subspace

$$
U_{j}^{I I I}(\mathbf{v}):=\left\{\left(i d_{j} \otimes \varphi_{[j]}\right)(\mathbf{v}): \varphi_{[j]} \in{ }_{a} \bigotimes_{k \neq j} V_{k}^{*}\right\}
$$

Finally, if $\mathbf{V}_{[j]}={ }_{a} \bigotimes_{k \neq j} V_{k}$ is a normed space for each $1 \leq j \leq d$, we can define

$$
U_{j}^{I V}(\mathbf{v}):=\left\{\left(i d_{j} \otimes \varphi_{[j]}\right)(\mathbf{v}): \varphi_{[j]} \in\left({ }_{a} \bigotimes_{k \neq j} V_{k}\right)^{*}\right\}
$$

Note that, in general, the four spaces ${ }_{a} \bigotimes_{k \neq j} V_{k}^{\prime},\left({ }_{a} \bigotimes_{k \neq j} V_{k}\right)^{\prime},{ }_{a} \bigotimes_{k \neq j} V_{k}^{*},\left({ }_{a} \bigotimes_{k \neq j} V_{k}\right)^{*}$ may differ.

Theorem 3.15 For any $\mathbf{v} \in \mathbf{V}={ }_{a} \bigotimes_{j=1}^{d} V_{j}$, there exist minimal subspaces $U_{j, \min }(\mathbf{v})(1 \leq j \leq d)$, whose algebraic characterisation is given by

$$
U_{j, \min }(\mathbf{v})=U_{j}^{I}(\mathbf{v})=U_{j}^{I I}(\mathbf{v}) .
$$

Furthermore, if $V_{j}$ and $\mathbf{V}_{[j]}={ }_{a} \bigotimes_{k \neq j} V_{k}$ are normed spaces for $1 \leq j \leq d$, then

$$
U_{j, \min }(\mathbf{v})=U_{j}^{I}(\mathbf{v})=U_{j}^{I I}(\mathbf{v})=U_{j}^{I I I}(\mathbf{v})=U_{j}^{I V}(\mathbf{v}) .
$$

Moreover, $\mathbf{v} \in{ }_{a} \bigotimes_{j=1}^{d} U_{j, \min }(\mathbf{v})$ and $\operatorname{dim}\left(U_{j, \min }(\mathbf{v})\right)=\operatorname{rank}_{j}(\mathbf{v})$ holds with $\operatorname{rank}_{j}$ from (3.6). 
Proof. Since the mappings $i d_{j} \otimes \varphi_{[j]}$ are applied to $\mathbf{v} \in \mathbf{U}:={ }_{a} \bigotimes_{j=1}^{d} U_{j, \min }(\mathbf{v})$, only the restrictions $\boldsymbol{\varphi}_{[j]}$ to ${ }_{a} \bigotimes_{k \neq j} U_{k, \min }(\mathbf{v})^{\prime}$ and $\left({ }_{a} \bigotimes_{k \neq j} U_{k, \min }(\mathbf{v})\right)^{\prime}$ are of interest. Since the subspace $U_{k, \min }(\mathbf{v})$, for all $k$, has finite dimension, Remark 3.4c states that $\left.{ }_{a} \bigotimes_{k \neq j} U_{k, \min }(\mathbf{v})^{\prime}={ }_{a} \bigotimes_{k \neq j} U_{j, \min }(\mathbf{v})\right)^{\prime}$. This proves $U_{j}^{I}(\mathbf{v})=U_{j}^{I I}(\mathbf{v})$.

Clearly, $U_{j}^{I I}(\mathbf{v}) \subset U_{j}^{I}(\mathbf{v})$. Now, let $u_{i}(1 \leq i \leq N)$ be a basis of $U_{j}^{I}(\mathbf{v})$. Since $\mathbf{v} \in{ }_{a} \bigotimes_{j=1}^{d} U_{j, \min }(\mathbf{v})$, there are $\varphi_{[j]}^{(i)}=\bigotimes_{k \neq j} \varphi_{k}^{(i)}$ with $\varphi_{k}^{(1)} \in U_{k, \min }(\mathbf{v})^{\prime}=U_{k, \min }(\mathbf{v})^{*}$ (note that the minimal subspaces are finite dimensional) and $u_{i}=\varphi_{[j]}^{(i)}(\mathbf{v})$. By the Hahn-Banach Theorem, the algebraic functionals $\varphi_{k}^{(i)}$ can be extended to $V_{k}$ such that $\varphi_{k}^{(i)} \in V_{k}^{*}$. This proves $U_{j}^{I}(\mathbf{v}) \subset U_{j}^{I I I}(\mathbf{v})$ and, in consequence, equality holds.

Next, we show that $U_{j}^{I I}(\mathbf{v})=U_{j}^{I V}(\mathbf{v})$. The inclusion $\left({ }_{a} \bigotimes_{k \neq j} V_{k}\right)^{*} \subset\left({ }_{a} \bigotimes_{k \neq j} V_{k}\right)^{\prime}$ implies $U_{j}^{I V}(\mathbf{v}) \subset$ $U_{j}^{I I}(\mathbf{v})$. Consider $v_{j}:=\left(i d_{j} \otimes \varphi_{[j]}\right)(\mathbf{v}) \in U_{j}^{I I}(\mathbf{v})$ for some $\varphi_{[j]} \in\left({ }_{a} \bigotimes_{k \neq j} V_{k}\right)^{\prime}$. Since $\mathbf{v} \in{ }_{a} \bigotimes_{j=1}^{d} U_{j, \min }(\mathbf{v})$, we may restrict $\boldsymbol{\varphi}_{[j]}$ to $\varphi_{[j]} \in\left({ }_{a} \bigotimes_{k \neq j} U_{k, \min }(\mathbf{v})\right)^{\prime}$. Since ${ }_{a} \bigotimes_{k \neq j} U_{k, \min }(\mathbf{v})$ is a finite dimensional subspace of the normed space ${ }_{a} \bigotimes_{k \neq j} V_{k}$, by the Hahn-Banach Theorem, the algebraic functional $\varphi_{[j]}$ can be extended to ${ }_{a} \bigotimes_{k \neq j} V_{k}$ such that $\overline{\varphi_{[j]}} \in\left({ }_{a} \bigotimes_{k \neq j} V_{k}\right)^{*}$, and by $v_{j}=\left(i d_{j} \otimes \overline{\varphi_{[j]}}\right)(\mathbf{v}) \in U_{j}^{I V}(\mathbf{v})$ the opposite inclusion $U_{j}^{I I}(\mathbf{v}) \subset U_{j}^{I V}(\mathbf{v})$ follows.

To prove that these space coincide with $U_{j, \min }(\mathbf{v})$, we apply the matricisation from $\S 3.1 .2$. The isomorphism $\mathcal{M}_{j}$ from Definition 3.5 maps ${ }_{a} \bigotimes_{k=1}^{d} V_{k}$ into $V_{j} \otimes_{a} V_{[j]}$ (cf. (3.3a)). Proposition 3.12 states that $U_{j, \min }(\mathbf{v})=U_{j}^{I I}(\mathbf{v})$ is the minimal subspace. So far, we have proved $\mathbf{v} \in U_{j, \min }(\mathbf{v}) \otimes_{a} V_{[j]}$. Thanks to Lemma 3.14, the intersection over all $1 \leq j \leq d$ yields $\mathbf{v} \in{ }_{a} \bigotimes_{j=1}^{d} U_{j, \min }(\mathbf{v})$.

Remark 3.16 While for $d=2, \operatorname{dim}\left(U_{1, \min }(\mathbf{v})\right)=\operatorname{dim}\left(U_{2, \min }(\mathbf{v})\right)$ holds, the dimensions of $U_{j, \min }(\mathbf{v})$ may be different for $d \geq 3$.

Since $U_{j, \min }(\mathbf{v})$ is a subspace of $V_{j}$ generated by elementary tensors from

$$
{ }_{a} \bigotimes_{k \neq j} V_{k}^{\prime}=\operatorname{span}\left\{\left(\varphi_{1} \otimes \cdots \otimes \varphi_{j-1} \otimes \varphi_{j+1} \otimes \cdots \otimes \varphi_{d}\right): \varphi_{k} \in V_{k}^{\prime}, k \neq j\right\}
$$

for $1 \leq j \leq d$, we can write

$$
U_{j, \min }(\mathbf{v})=\operatorname{span}\left\{\left(\varphi_{1} \otimes \cdots \otimes \varphi_{j-1} \otimes i d_{j} \otimes \varphi_{j+1} \otimes \cdots \otimes \varphi_{d}\right)(\mathbf{v}): \varphi_{k} \in V_{k}^{\prime}, k \neq j\right\},
$$

and, if $V_{k}$ is a normed space for $1 \leq k \leq d$, also as

$$
U_{j, \min }(\mathbf{v})=\operatorname{span}\left\{\left(\varphi_{1} \otimes \cdots \otimes \varphi_{j-1} \otimes i d_{j} \otimes \varphi_{j+1} \otimes \cdots \otimes \varphi_{d}\right)(\mathbf{v}): \varphi_{k} \in V_{k}^{*}, k \neq j\right\}
$$

for $1 \leq j \leq d$.

\subsubsection{Hierarchies of minimal subspaces}

We have introduced the minimal subspace $U_{j, \min }(\mathbf{v}) \subset V_{j}$ for a singleton $\{j\} \subset \mathcal{I}=\{1,2, \ldots, d\}$. Instead we may consider general disjoint and non-empty subsets of $\mathcal{I}$. For instance, let $\mathbf{v} \in{ }_{a} \otimes_{j \in \mathcal{J}} V_{j}=V_{\mathcal{J}_{1}} \otimes V_{\mathcal{J}_{2}} \otimes V_{\mathcal{J}_{3}}$, where $\mathcal{J}_{1}=\{1,2\}, \mathcal{J}_{2}=\{3,4\}$, and $\mathcal{J}_{3}=\{5,6,7\}$. Then we can conclude that there are minimal subspaces $U_{\mathcal{J}_{j}, \min }(\mathbf{v})$ for $j=1,2,3$, such that

$$
\mathbf{v} \in{ }_{a} \bigotimes_{j=1}^{3} U_{\mathcal{J}_{j}, \min }(\mathbf{v})
$$

The relation between $U_{j, \min }(\mathbf{v})$ and $U_{\mathcal{J}_{j}, \min }(\mathbf{v})$ is as follows.

Proposition 3.17 Let $\mathbf{v} \in \mathbf{V}={ }_{a} \bigotimes_{j \in \mathcal{I}} V_{j}$ and $\emptyset \neq \mathcal{J} \subset \mathcal{I}$. Then the minimal subspaces $U_{\mathcal{J}, \min }(\mathbf{v})$ and $U_{j, \min }(\mathbf{v})$ for $j \in \mathcal{J}$ are related by

$$
U_{\mathcal{J}, \min }(\mathbf{v}) \subset{ }_{a} \bigotimes_{j \in \mathcal{J}} U_{j, \min }(\mathbf{v}) .
$$


Proof. We know that $\mathbf{v} \in \mathbf{U}={ }_{a} \bigotimes_{j \in \mathcal{I}} U_{j, \min }(\mathbf{v})$. We may write $\mathbf{U}=\mathbf{U}_{\mathcal{J}} \otimes_{a} \mathbf{U}_{\mathcal{J}^{c}}$, where $\mathcal{J}^{c}=\mathcal{I} \backslash \mathcal{J}$ and $\mathbf{U}_{\mathcal{K}}:={ }_{a} \bigotimes_{j \in \mathcal{K}} U_{j, \min }(\mathbf{v})$ for any subset $\mathcal{K} \subset \mathcal{I}$. Thus, $U_{\mathcal{J}, \text { min }}(\mathbf{v})$ must be contained in $\mathbf{U}_{\mathcal{J}}=$ ${ }_{a} \bigotimes_{j \in \mathcal{J}} U_{j, \min }(\mathbf{v})$.

An obviously generalisation of the previous results is given below.

Corollary 3.18 Let $\mathbf{v} \in \mathbf{V}={ }_{a} \bigotimes_{j \in \mathcal{I}} V_{j}$. Assume that $\emptyset \neq \mathcal{J}_{j} \subset \mathcal{I}$ are pairwise disjoint for $j=1,2, \ldots, m$. The minimal subspace for $\mathcal{J}:=\bigcup_{j=1}^{m} \mathcal{J}_{j}$ satisfies

$$
U_{\mathcal{J}, \min }(\mathbf{v}) \subset{ }_{a} \bigotimes_{i=1}^{m} U_{\mathcal{J}_{i}, \min }(\mathbf{v})
$$

The algebraic characterisation of $U_{\mathcal{J}, \min }(\mathbf{v})$ is analogous to that given in Theorem 3.15. Formulae (3.8a,b) become

$$
\begin{aligned}
U_{\mathcal{J}, \min }(\mathbf{v}) & =\left\{\left(i d_{\mathcal{J}} \otimes \boldsymbol{\varphi}_{\mathcal{J}^{c}}\right)(\mathbf{v}): \boldsymbol{\varphi}_{\mathcal{J}^{c}} \in{ }_{a} \bigotimes_{j \in \mathcal{J}^{c}} V_{j}^{\prime}\right\} \\
& =\left\{\left(i d_{\mathcal{J}} \otimes \boldsymbol{\varphi}_{\mathcal{J}^{c}}\right)(\mathbf{v}): \boldsymbol{\varphi}_{\mathcal{J}^{c}} \in\left(a \bigotimes_{j \in \mathcal{J}^{c}} V_{j}\right)^{\prime}\right\},
\end{aligned}
$$

where $\left(i d_{\mathcal{J}} \otimes_{\mathcal{J}^{c}}\right)\left(\otimes_{j=1}^{d} v_{j}\right)=\left(\varphi_{\mathcal{J}^{c}}\left(\otimes_{j \in \mathcal{J}^{c}} v_{j}\right)\right) \otimes_{k \in \mathcal{J}} v_{k}$. The analogues of $(3.8 c, d)$ apply as soon as norms are defined on $V_{j}$ and ${ }_{a} \bigotimes_{j \in \mathcal{J}^{c}} V_{j}$.

\section{Minimal subspaces in a Banach tensor space}

In this section we assume the existence of a norm, namely $\|\cdot\|$, defined on a tensor space V. More precisely, we introduce the following class of Banach spaces.

Definition 4.1 We say that $\mathbf{V}_{\|\cdot\|}$ is a Banach tensor space if there exists an algebraic tensor space $\mathbf{V}$ and a norm $\|\cdot\|$ on $\mathbf{V}$ such that $\mathbf{V}_{\|\cdot\|}$ is the completion of $\mathbf{V}$ with respect a given norm $\|\cdot\|$, i.e.,

$$
\mathbf{V}_{\|\cdot\|}:=\|\cdot\| \bigotimes_{j=1}^{d} V_{j}=\bar{a}_{\bigotimes_{j=1}^{d} V_{j}}^{\|\cdot\|}
$$

If $\mathbf{V}_{\|\cdot\|}$ is a Hilbert space, we will say that $\mathbf{V}_{\|\cdot\|}$ is a Hilbert tensor space.

Next, we give some examples of Banach and Hilbert tensor spaces.

Example 4.2 For $I_{j} \subset \mathbb{R}(1 \leq j \leq d)$ and $1 \leq p<\infty$, the Sobolev space $H^{N, p}\left(I_{j}\right)$ consists of all function $f$ from $L^{p}\left(I_{j}\right)$ with bounded norm ${ }^{1}$

$$
\|f\|_{N, p ; I_{j}}:=\left(\sum_{n=0}^{N} \int_{I_{j}}\left|\frac{d^{n}}{d x^{n}} f\right|^{p} \mathrm{~d} x\right)^{1 / p},
$$

whereas the space $H^{N, p}(\mathbf{I})$ of d-variate functions on $\mathbf{I}=I_{1} \times I_{2} \times \ldots \times I_{d} \subset \mathbb{R}^{d}$ is endowed with the norm

$$
\|f\|_{N, p}:=\left(\sum_{0 \leq|\mathbf{n}| \leq N} \int_{\mathbf{I}}\left|\partial^{\mathbf{n}} f\right|^{p} \mathrm{~d} \mathbf{x}\right)^{1 / p}
$$

with $\mathbf{n} \in \mathbb{N}_{0}^{d}$ being a multi-index of length $|\mathbf{n}|:=\sum_{j=1}^{d} n_{j}$. It is well-known that $H^{N, p}\left(I_{j}\right)$ and $H^{N, p}(\mathbf{I})$ are reflexive and separable Banach spaces. Moreover, for $p=2$, the Sobolev spaces $H^{N}\left(I_{j}\right):=H^{N, 2}\left(I_{j}\right)$ and $H^{N}(\mathbf{I}):=H^{N, 2}(\mathbf{I})$ are Hilbert spaces. As a first example,

$$
H^{N, p}(\mathbf{I})=\|_{\|\cdot\|_{N, p}} \bigotimes_{j=1}^{d} H^{N, p}\left(I_{j}\right)
$$

\footnotetext{
${ }^{1}$ It suffices to have in (4.1a) the terms for $n=0$ and $n=N$. The derivatives are to be understood as weak derivatives.
} 
is a Banach tensor space. Examples of Hilbert tensor spaces are

$$
L^{2}(\mathbf{I})=\|\cdot\|_{0,2} \bigotimes_{j=1}^{d} L^{2}\left(I_{j}\right) \quad \text { and } \quad H^{N}(\mathbf{I})=\|\cdot\|_{N, 2} \bigotimes_{j=1}^{d} H^{N}\left(I_{j}\right) \text { for } N \in \mathbb{N} .
$$

We recall that for the set of norms over a given vector space $V$, we can define a partial ordering $\|\cdot\|_{1} \lesssim\|\cdot\|_{2}$ (in words: $\|\cdot\|_{1}$ weaker than $\|\cdot\|_{2}$ ), if there exists a constant $C$ such that $\|v\|_{1} \leq C\|v\|_{2}$ for all $v \in V$.

Given a vector space $V$, its completion with respect to a norm $\|\cdot\|$ yields a Banach space which we denote by $V_{\|\cdot\|}:=\bar{V}^{\|\cdot\|}$. Note that $\|\cdot\|_{1} \lesssim\|\cdot\|_{2}$ implies that $V_{\|\cdot\|_{2}} \subset V_{\|\cdot\|_{1}}$.

\subsection{Tensor product of Banach spaces}

Let $\|\cdot\|_{j}, 1 \leq j \leq d$, be the norms of the vector spaces $V_{j}$ appearing in $\mathbf{V}={ }_{a} \bigotimes_{j=1}^{d} V_{j}$. By $\|\cdot\|$ we denote the norm on the tensor space $\mathbf{V}$. Note that $\|\cdot\|$ is not determined by $\|\cdot\|_{j}$, but there are relations which are 'reasonable'.

Any norm $\|\cdot\|$ on ${ }_{a} \bigotimes_{j=1}^{d} V_{j}$ satisfying

$$
\left\|\bigotimes_{j=1}^{d} v^{(j)}\right\|=\prod_{j=1}^{d}\left\|v^{(j)}\right\|_{j} \quad \text { for all } v^{(j)} \in V_{j}(1 \leq j \leq d)
$$

is called a crossnorm. As usual, the dual norm to $\|\cdot\|$ is denoted by $\|\cdot\|^{*}$. If $\|\cdot\|$ is a crossnorm and also $\|\cdot\|^{*}$ is a crossnorm on ${ }_{a} \bigotimes_{j=1}^{d} V_{j}^{*}$, i.e.,

$$
\left\|\bigotimes_{j=1}^{d} \varphi^{(j)}\right\|^{*}=\prod_{j=1}^{d}\left\|\varphi^{(j)}\right\|_{j}^{*} \quad \text { for all } \varphi^{(j)} \in V_{j}^{*}(1 \leq j \leq d),
$$

$\|\cdot\|$ is called a reasonable crossnorm.

Remark 4.3 Eq. (4.2) implies the inequality $\left\|\bigotimes_{j=1}^{d} v^{(j)}\right\| \lesssim \prod_{j=1}^{d}\left\|v^{(j)}\right\|_{j}$ which is equivalent to the continuity of the tensor product mapping

$$
\bigotimes: \underset{j=1}{\underset{X}{X}}\left(V_{j},\|\cdot\|_{j}\right) \longrightarrow\left({ }_{a} \bigotimes_{j=1}^{d} V_{j},\|\cdot\|\right)
$$

given by $\otimes\left(\left(v_{1}, \ldots, v_{d}\right)\right)=\otimes_{j=1}^{d} v_{j}$.

By standard arguments, continuity of the tensor product implies the following result.

Lemma 4.4 Let $V_{j, 0}$ be dense in Banach spaces $\left(V_{j},\|\cdot\|_{j}\right)$ for $1 \leq j \leq d$. Assume (4.4) to be continuous for

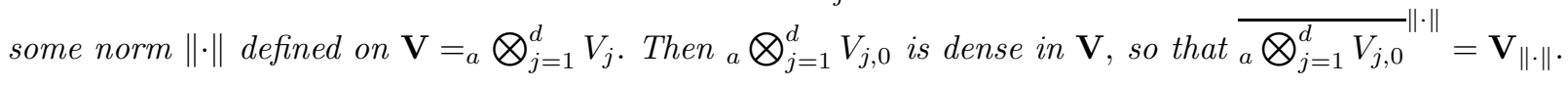

Example 4.5 It is well-known that the norm $\|\cdot\|_{0,2}$ is a reasonable crossnorm on a $\bigotimes_{j=1}^{d} L^{2}\left(I_{j}\right)$, whereas $\|\cdot\|_{N, 2}$ for $N \geq 1$ not is a reasonable crossnorm on ${ }_{a} \bigotimes_{j=1}^{d} H^{N}\left(I_{j}\right)$ (cf. Example 4.2).

Note that any functional $\varphi=\otimes_{j=1}^{d} \varphi_{j} \in{ }_{a} \bigotimes_{j=1}^{d} V_{j}^{*}$ is also a linear map ${ }_{a} \bigotimes_{j=1}^{d} V_{j} \rightarrow \mathbb{R}$, which is defined for elementary tensors by

$$
\left(\bigotimes_{j=1}^{d} \varphi_{j}\right)\left(\otimes_{j=1}^{d} v_{j}\right)=\prod_{j=1}^{d} \varphi_{j}\left(v_{j}\right)
$$

Thus, ${ }_{a} \bigotimes_{j=1}^{d} V_{j}^{*} \subset\left({ }_{a} \bigotimes_{j=1}^{d} V_{j}\right)^{\prime}$, because continuity is not ensured. If $\|\cdot\|$ is a reasonable crossnorm, then by (4.3) the map

$$
\bigotimes: \underset{j=1}{\mathbb{X}}\left(V_{j}^{*},\|\cdot\|_{j}^{*}\right) \longrightarrow\left({ }_{a} \bigotimes_{j=1}^{d} V_{j}^{*},\|\cdot\|^{*}\right),
$$

is also continuous. In consequence, ${ }_{a} \bigotimes_{j=1}^{d} V_{j}^{*} \subset\left({ }_{a} \bigotimes_{j=1}^{d} V_{j}\right)^{*}$.

Grothendieck [11] named the following norm $\|\cdot\|_{\vee}$ the injective norm. 
Definition 4.6 Let $V_{i}$ be a Banach spaces with norm $\|\cdot\|_{i}$ for $1 \leq i \leq d$. Then for $\mathbf{v} \in \mathbf{V}={ }_{a} \bigotimes_{j=1}^{d} V_{j}$ define $\|\cdot\|_{\vee}$ by

$$
\|\mathbf{v}\|_{\vee}:=\sup \left\{\frac{\left|\left(\varphi_{1} \otimes \varphi_{2} \otimes \ldots \otimes \varphi_{d}\right)(\mathbf{v})\right|}{\prod_{j=1}^{d}\left\|\varphi_{j}\right\|_{j}^{*}}: 0 \neq \varphi_{j} \in V_{j}^{*}, 1 \leq j \leq d\right\} .
$$

It can be shown that the injective norm is a reasonable crossnorm. Further properties are given by the next proposition.

Proposition 4.7 The following statements hold.

(a) The injective norm is the weakest reasonable crossnorm on $\mathbf{V}$, i.e., if $\|\cdot\|$ is a reasonable crossnorm over $\mathbf{V}$, then $\|\cdot\|_{\vee} \lesssim\|\cdot\|$.

(b) For any norm $\|\cdot\|$ on $\mathbf{V}$ satisfying $\|\cdot\|_{\vee} \lesssim\|\cdot\|$, the inclusion ${ }_{a} \bigotimes_{j=1}^{d} V_{j}^{*} \subset\left({ }_{a} \bigotimes_{j=1}^{d} V_{j}\right)^{*}$ holds.

Proof. Statement (a) is a classical result (cf. [16]). To prove (b), we use that $\|\cdot\|_{\vee} \lesssim\|\cdot\|$ implies that $\|\cdot\|_{\vee}^{*} \gtrsim\|\cdot\|^{*}$ (see again $[16]$ ). Then

$$
\left\|\otimes_{j=1}^{d} \varphi_{j}\right\|^{*} \leq C\left\|\otimes_{j=1}^{d} \varphi_{j}\right\|_{\vee}^{*} \quad\left(\varphi_{j} \in V_{j}^{*}, 1 \leq j \leq d\right)
$$

for some $C>0$ and the proof ends using that $\|\cdot\|_{v}^{*}$ is also a crossnorm.

\subsection{Minimal subspaces in a Banach tensor space}

Let $\mathbf{V}$ be a tensor product of Banach spaces $\left(V_{i},\|\cdot\|_{i}\right)$ for $1 \leq i \leq d$. Then, considering the injective norm on $\mathbf{V}_{[j]}$ for $1 \leq j \leq d$, for each $\mathbf{v} \in \mathbf{V}$, we conclude from Theorem 3.15 that $^{2}$

$$
U_{j, \min }(\mathbf{v})=U_{j}^{I}(\mathbf{v})=U_{j}^{I I}(\mathbf{v})=U_{j}^{I I I}(\mathbf{v})=U_{j}^{I V}(\mathbf{v})
$$

(cf. (3.8a-d)). Assume that there exists a norm $\|\cdot\|$ on $\mathbf{V}$ satisfying

$$
\|\cdot\| \gtrsim\|\cdot\|_{\vee}
$$

(cf. Proposition 4.7a). This assumption ensures that the Banach tensor space $\mathbf{V}_{\|\cdot\|}$ is always a Banach subspace of the Banach tensor space $\mathbf{V}_{\|\cdot\|_{\vee}}$. This fact allows to extend the definition of minimal subspaces to a Banach tensor space $\mathbf{V}_{\|\cdot\|}$ with a norm $\|\cdot\|$ satisfying (4.6). To this end, the following lemma will be useful.

Lemma 4.8 For $1 \leq i \leq d$, let $\left(V_{i},\|\cdot\|_{i}\right)$ be a Banach space. For fixed $j \in\{1, \ldots, d\}$ and a given $\varphi_{[j]}=$ $\bigotimes_{k \neq j} \varphi_{k} \in{ }_{a} \bigotimes_{k \neq j} V_{k}^{*}$, the map $i d_{j} \otimes \varphi_{[j]}$ belongs not only to $L\left(\mathbf{V}, V_{j}\right)$ but also to $\mathcal{L}\left(\mathbf{V}, V_{j}\right)$, i.e., id $\otimes_{j} \otimes \boldsymbol{\varphi}_{[j]}$ is continuous on $\left(\mathbf{V},\|\cdot\|_{\vee}\right)$. Hence, there exists a unique extension $\overline{i d_{j} \otimes \varphi_{[j]}} \in \mathcal{L}\left(\mathbf{V}_{\|\cdot\|_{\vee}}, V_{j}\right)$. Moreover, $\overline{i d_{j} \otimes \varphi_{[j]}} \in \mathcal{L}\left(\mathbf{V}_{\|\cdot\|}, V_{j}\right)$ holds for any norm $\|\cdot\|$ on $\mathbf{V}$ satisfying (4.6) with the operator norm

$$
\left\|\overline{i d_{j} \otimes \varphi_{[j]}}\right\|_{V_{j} \leftarrow \mathbf{v}}=\sup _{\|\mathbf{v}\|=1}\left\|\left(\overline{i d_{j} \otimes \varphi_{[j]}}\right)(\mathbf{v})\right\|_{j} \leq C \prod_{k \neq j}\left\|\varphi_{k}\right\|_{k}^{*}
$$

where the constant $C$ is determined by the estimate in (4.6).

Proof. Let $\varphi_{j} \in V_{j}^{*}$ and use $\varphi_{j} \circ\left(i d_{j} \otimes \varphi_{[j]}\right)=\bigotimes_{k=1}^{d} \varphi_{k}$ (cf. (3.5f)). Hence, continuity follows from

$$
\begin{aligned}
\left\|\left(i d_{j} \otimes \varphi_{[j]}\right)(\mathbf{v})\right\|_{j} & \underset{(2.2)}{=} \max _{\varphi_{j} \in V_{j}^{*},\left\|\varphi_{j}\right\|_{j}^{*}=1}\left|\left(\varphi_{j} \circ\left(i d_{j} \otimes \varphi_{[j]}\right)\right)(\mathbf{v})\right| \\
& =\max _{\left\|\varphi_{j}\right\|_{j}^{*}=1}\left|\left(\bigotimes_{k=1}^{d} \varphi_{k}\right)(\mathbf{v})\right| \underset{(4.3)}{\leq}\left(\prod_{k \neq j}\left\|\varphi_{k}\right\|_{k}^{*}\right)\|\mathbf{v}\|_{v} \\
& \leq C\left(\prod_{k \neq j}\left\|\varphi_{k}\right\|_{k}^{*}\right)\|\mathbf{v}\| .
\end{aligned}
$$

\footnotetext{
${ }^{2}$ We recall that the definition of $U_{j}^{I V}(\mathbf{v})$ requires the definition of a norm on $\mathbf{V}_{[j]}$. The following arguments will be based on $U_{j}^{I I I}(\mathbf{v})$.
} 
The last inequality holds for any norm on $\mathbf{V}$ satisfying $\|\cdot\| \geq(1 / C)\|\cdot\|_{\vee}$ and proves (4.7). The statement about the extension $\overline{i d_{j} \otimes \varphi_{[j]}}$ is standard.

An immediate consequence of Lemma 4.8 and Theorem 3.15 is the following.

Corollary 4.9 For $1 \leq i \leq d$, let $\left(V_{i},\|\cdot\|_{i}\right)$ be a Banach space and assume that $\|\cdot\|$ is a norm on $\mathbf{V}$ satisfying (4.6). Then for each algebraic tensor $\mathbf{v} \in \mathbf{V}$ the representation

$$
U_{j, \min }(\mathbf{v})=\left\{\left(\overline{i d_{j} \otimes \boldsymbol{\varphi}_{[j]}}\right)(\mathbf{v}): \boldsymbol{\varphi}_{[j]} \in \bigotimes_{k \neq j} V_{k}^{*}\right\}
$$

holds for $1 \leq j \leq d$. Moreover, we can write

$$
U_{j, \min }(\mathbf{v})=\operatorname{span}\left\{\left(\overline{\varphi_{1} \otimes \cdots \otimes \varphi_{j-1} \otimes i d_{j} \otimes \varphi_{j+1} \otimes \cdots \otimes \varphi_{d}}\right)(\mathbf{v}): \varphi_{k} \in V_{k}^{*}, k \neq j\right\} .
$$

For the hierarchical format from [12] we need to extend the results to a minimal subspace in the tensor space $\mathbf{V}_{\mathcal{J}}:=\bigotimes_{k \in \mathcal{J}} V_{k}$, where $\mathcal{J} \subset\{1, \ldots, d\}$ contains more than one index. Then the splitting $V_{j} \otimes \mathbf{V}_{[j]}$ from above becomes $\mathbf{V}_{\mathcal{J}} \otimes \mathbf{V}_{\mathcal{J}^{c}}$, where $\mathbf{V}_{\mathcal{J}^{c}}:=\bigotimes_{k \in\{1, \ldots, d\} \backslash \mathcal{J}} V_{k}$. The definition of, e.g., $U_{j}^{I}(\mathbf{v})$ in (3.8a) becomes

$$
\mathbf{U}_{\mathcal{J}}^{I}(\mathbf{v}):=\left\{\left(i d_{\mathcal{J}} \otimes \boldsymbol{\varphi}_{\mathcal{J}^{c}}\right)(\mathbf{v}): \boldsymbol{\varphi}_{\mathcal{J}^{c}} \in{ }_{a} \bigotimes_{k \in \mathcal{J}} V_{k}^{\prime}\right\} \subset \mathbf{V}_{\mathcal{J}}
$$

involving the identity $i d_{\mathcal{J}} \in L\left(\mathbf{V}_{\mathcal{J}}, \mathbf{V}_{\mathcal{J}}\right)$.

Remark 4.10 By analogous arguments as above, we can show

$$
\mathbf{U}_{\mathcal{J}}^{I}(\mathbf{v})=\mathbf{U}_{\mathcal{J}}^{I I I}(\mathbf{v}):=\left\{\left(i d_{\mathcal{J}} \otimes \boldsymbol{\varphi}_{\mathcal{J}^{c}}\right)(\mathbf{v}): \varphi_{\mathcal{J}^{c}} \in{ }_{a} \bigotimes_{k \in \mathcal{J}} V_{k}^{*}\right\} .
$$

In particular, $\overline{\text { id } d_{\mathcal{J}} \otimes \varphi_{\mathcal{J}^{c}}} \in \mathcal{L}\left(\mathbf{V}_{\|\cdot\|_{\vee}}, V_{\mathcal{J}}\right) \subset \mathcal{L}\left(\mathbf{V}_{\|\cdot\|}, V_{\mathcal{J}}\right)$ holds.

\subsection{Minimal closed subspaces in a Banach tensor space}

\subsubsection{Definitions}

So far, $U_{j \min }(\mathbf{v})$ has been defined for algebraic tensors only. Because of $\overline{i d_{j} \otimes \varphi_{[j]}} \in \mathcal{L}\left(\mathbf{V}_{\|\cdot\|}, V_{j}\right)$, we can extend the definition of $U_{j \min }(\mathbf{v})$ in Corollary 4.9 even to topological tensors $\mathbf{v} \in \mathbf{V}_{\|\cdot\|} \backslash \mathbf{V}$ as follows.

Definition 4.11 For a given Banach tensor space $\mathbf{V}_{\|\cdot\|}$ with a norm $\|\cdot\|$ satisfying (4.6) we define the set

$$
U_{j, \min }(\mathbf{v}):=\left\{\left(\overline{i d_{j} \otimes \boldsymbol{\varphi}_{[j]}}\right)(\mathbf{v}): \boldsymbol{\varphi}_{[j]} \in a \bigotimes_{k \neq j} V_{k}^{*}\right\}
$$

for each $\mathbf{v} \in \mathbf{V}_{\|\cdot\|}$ and $1 \leq j \leq d$.

Observe that $\left(\overline{i d_{j} \otimes \varphi_{[j]}}\right)(\mathbf{v})$ is well-defined, because $\overline{i d_{j} \otimes \varphi_{[j]}}$ is continuous and coincides with the standard definition when $\mathbf{v} \in \mathbf{V}$. Thus, for each $\mathbf{v} \in \mathbf{V}_{\|\cdot\|}$ we can define its 'minimal subspace' by

$$
\mathbf{U}(\mathbf{v}):={ }_{a} \bigotimes_{j=1}^{d} U_{j, \min }(\mathbf{v}) .
$$

If we take into account the topological properties of $\mathbf{V}_{\|\cdot\|}$, we may consider its closure with respect the norm $\|\cdot\|$ :

$$
\mathbf{U}_{\|\cdot\|}(\mathbf{v}):=\|\cdot\| \bigotimes_{j=1}^{d} \overline{U_{j, \min }(\mathbf{v})}\|\cdot\|_{j}=\|\cdot\| \bigotimes_{j=1}^{d} U_{j, \min }(\mathbf{v}) .
$$

The second identity is a consequence of Lemma 4.4. If $\mathbf{v} \in \mathbf{V}$, the set $U_{j, \min }(\mathbf{v})$ is a finite dimensional subspace in $V_{j}$ and therefore closed, i.e., ${\overline{U_{j, \min }(\mathbf{v})}}^{\|\cdot\|_{j}}=U_{j, \min }(\mathbf{v})$. In the general case of $\mathbf{v} \in \mathbf{V}_{\|\cdot\|}$, the subspace $U_{j, \min }(\mathbf{v})$ may be not closed.

Before we discuss the Banach subspace $\mathbf{U}_{\|\cdot\|}(\mathbf{v})$ in $\S 4.3 .3$, we first analyse the properties of the subspace $U_{j, \min }(\mathbf{v})$. 


\subsubsection{Dependence of $U_{j, \min }(\mathbf{v})$ on $\mathbf{v}$}

The properties of the maps $i d_{j} \otimes \boldsymbol{\varphi}_{[j]}$ involved in the definition of $U_{j, \min }(\mathbf{v})$ are discussed in Lemma 4.12. As a consequence, we shall establish our main result in Theorem 4.13 about the dimensions of $U_{j, \min }\left(\mathbf{v}_{n}\right)$ and $U_{j, \min }(\mathbf{v})$ for a weakly convergent sequence $\mathbf{v}_{n} \rightarrow \mathbf{v}$.

Lemma 4.12 Assume that the norm of the Banach tensor space $\mathbf{V}_{\|\cdot\|}$ satisfies (4.6). Let $\varphi_{[j]} \in{ }_{a} \bigotimes_{k \neq j} V_{k}^{*}$ and $\mathbf{v}_{n}, \mathbf{v} \in \mathbf{V}_{\|\cdot\|}$ with $\mathbf{v}_{n} \rightarrow \mathbf{v}$. Then weak convergence $\left(\overline{i d_{j} \otimes \varphi_{[j]}}\right)\left(\mathbf{v}_{n}\right) \rightarrow\left(\overline{i d_{j} \otimes \varphi_{[j]}}\right)(\mathbf{v})$ holds in $V_{j}$.

Proof. Let $\varphi_{[j]}=\otimes_{k \neq j} \varphi_{k} \in{ }_{a} \bigotimes_{k \neq j} V_{k}^{*}$ be an elementary tensor. We have to show that

$$
\varphi_{j}\left[\left(\overline{i d_{j} \otimes \varphi_{[j]}}\right)\left(\mathbf{v}_{n}\right)\right] \rightarrow \varphi_{j}\left[\left(\overline{i d_{j} \otimes \varphi_{[j]}}\right)(\mathbf{v})\right]
$$

holds for all $\varphi_{j} \in V_{j}^{*}$. By Lemma 4.8, $\overline{i d_{j} \otimes \varphi_{[j]}}: \mathbf{V}_{\|\cdot\|} \rightarrow V_{j}$ is continuous. Therefore, the composition $\varphi_{j} \circ\left(\overline{i d_{j} \otimes \varphi_{[j]}}\right): \mathbf{V}_{\|\cdot\|} \rightarrow \mathbb{R}$ is a continuous functional belonging to $\mathbf{V}_{\|\cdot\|}^{*}$, and hence $\mathbf{v}_{n} \rightarrow \mathbf{v}$ implies

$$
\left(\varphi_{j} \circ\left(\overline{i d_{j} \otimes \varphi_{[j]}}\right)\right)\left(\mathbf{v}_{n}\right) \rightarrow\left(\varphi_{j} \circ\left(\overline{i d_{j} \otimes \varphi_{[j]}}\right)\right)(\mathbf{v}) .
$$

This proves the lemma for an elementary tensor $\varphi_{[j]}$. The result extends immediately to finite linear combinations $\varphi_{[j]} \in{ }_{a} \bigotimes_{k \neq j} V_{k}^{*}$.

Theorem 4.13 Assume that the norm of the Banach tensor space $\mathbf{V}_{\|\cdot\|}$ satisfies (4.6). Let $\mathbf{v}_{n} \in \mathbf{V}_{\|\cdot\|}$ be a sequence with $\mathbf{v}_{n} \rightarrow \mathbf{v} \in \mathbf{V}_{\|\cdot\| \cdot}$. Then ${ }^{3}$

$$
\operatorname{dim} \overline{U_{j, \min }(\mathbf{v})}\|\cdot\|_{j}=\operatorname{dim} U_{j, \min }(\mathbf{v}) \leq \liminf _{n \rightarrow \infty} \operatorname{dim} U_{j, \min }\left(\mathbf{v}_{n}\right) \quad \text { for all } 1 \leq j \leq d .
$$

Proof. Since $U_{j, \min }(\mathbf{v})$ is dense in $\overline{U_{j, \min }(\mathbf{v})}\|\cdot\|_{j}$, the dimensions are identical in the sense of Footnote 3. We can select a subsequence (again denoted by $\mathbf{v}_{n}$ ) such that $\operatorname{dim} U_{j, \min }\left(\mathbf{v}_{n}\right)$ is weakly increasing. If $\operatorname{dim} U_{j, \min }\left(\mathbf{v}_{n}\right) \rightarrow \infty$ holds, nothing is to be proved. Therefore, assume that $\lim \operatorname{dim} U_{j, \min }\left(\mathbf{v}_{n}\right)=N<\infty$. For an indirect proof assume that $\operatorname{dim} U_{j, \min }(\mathbf{v})>N$. Then, there are $N+1$ linearly independent vectors

$$
\left.b^{(i)}=\overline{\left(i d_{j} \otimes \varphi_{[j]}^{(i)}\right.}\right)(\mathbf{v}) \quad \text { with } \varphi_{[j]}^{(i)} \in{ }_{a} \bigotimes_{k \neq j} V_{k}^{*} \quad \text { for } 1 \leq i \leq N+1 .
$$

By Lemma 4.12, the sequence $\left.b_{n}^{(i)}:=\overline{\left(i d_{j} \otimes \boldsymbol{\varphi}_{[j]}^{(i)}\right.}\right)\left(\mathbf{v}_{n}\right) \rightarrow b^{(i)}$ converges weakly. By Lemma 2.5, for large enough $n$, also $\left\{b_{n}^{(i)}: 1 \leq i \leq N+1\right\}$ is linearly independent. Because of $\left.b_{n}^{(i)}=\overline{\left(i d_{j} \otimes \varphi_{[j]}^{(i)}\right.}\right)\left(\mathbf{v}_{n}\right) \in U_{j, \min }\left(\mathbf{v}_{n}\right)$, this contradicts $\operatorname{dim} U_{j, \min }\left(\mathbf{v}_{n}\right) \leq N$.

For the hierarchical format from [12], $i d_{j} \otimes \boldsymbol{\varphi}_{[j]}$ has to be replaced by $i d_{\mathcal{J}} \otimes \boldsymbol{\varphi}_{\mathcal{J}^{c}}$ (cf. Corollary 3.18 and Remark 4.10). Similar lines as above show the following generalisations:

$$
\begin{gathered}
\mathbf{v}_{n} \rightarrow \mathbf{v} \text { in } \mathbf{V} \Rightarrow\left(\overline{i d_{\mathcal{J}} \otimes \varphi_{\mathcal{J}^{c}}}\right)\left(\mathbf{v}_{n}\right) \rightarrow\left(\overline{i d_{\mathcal{J}} \otimes \varphi_{\mathcal{J}^{c}}}\right)(\mathbf{v}) \text { in }\|\cdot\|_{\vee} \bigotimes_{j \in \mathcal{J}} V_{j}, \\
\operatorname{dim} \overline{U_{\mathcal{J}, \min }(\mathbf{v})}\|\cdot\|_{\vee} \leq \liminf _{n \rightarrow \infty} \operatorname{dim} U_{\mathcal{J}, \min }\left(\mathbf{v}_{n}\right) \quad \text { for all } \emptyset \neq \mathcal{J} \subset\{1, \ldots, d\} .
\end{gathered}
$$

Here, we equip the tensor space $\mathbf{V}_{\mathcal{J}}={ }_{a} \bigotimes_{j \in \mathcal{J}} V_{j}$ with the injective norm $\|\cdot\|_{\vee}$ from (4.5).

4.3.3 $\operatorname{dim}\left(U_{j, \min }(\mathbf{v})\right)<\infty$

Consider $\mathbf{U}(\mathbf{v})$ and $\mathbf{U}_{\|\cdot\|}(\mathbf{v})$ from $(4.8 \mathrm{a}, \mathrm{b})$. For algebraic tensors $\mathbf{v}$ we know that $\mathbf{v} \in \mathbf{U}(\mathbf{v})$. However, the corresponding conjecture $\mathbf{v} \in \mathbf{U}_{\|\cdot\|}(\mathbf{v})$, in the general case, turns out to be not quite obvious. The statement

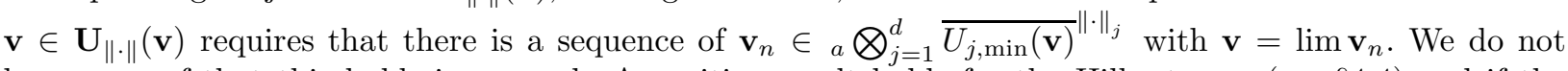
have a proof that this holds in general. A positive result holds for the Hilbert case (see §4.4) and if the

\footnotetext{
${ }^{3}$ Here, infinite dimensions are identified and not considered as possibly different infinite cardinalities.
} 
subspaces $U_{j, \min }(\mathbf{v})$ are finite dimensional (see Theorem 4.14). In the general Banach case, we give a proof for $\mathbf{v}=\lim \mathbf{v}_{n}$, provided that the convergence is fast enough.

For practical applications, the finite dimensional case is the most important one, since this follows from Theorem 4.13 with bounded $\lim \inf _{n \rightarrow \infty} \operatorname{dim} U_{j, \min }\left(\mathbf{v}_{n}\right)$.

Theorem 4.14 Assume that $\mathbf{V}_{\|\cdot\|}$ is a Banach tensor space with $\|\cdot\|$ satisfying (4.6). For $\mathbf{v} \in \mathbf{V}_{\|\cdot\|}$ and all $1 \leq j \leq d$ assume that $\operatorname{dim}\left(U_{j, \min }(\mathbf{v})\right)<\infty$. Then $\mathbf{v}$ belongs to the (algebraic) tensor space ${ }_{a} \bigotimes_{j=1}^{d} U_{j, \min }(\mathbf{v})=\mathbf{U}_{\|\cdot\|}(\mathbf{v})$.

Proof. Let $\left\{b_{j}^{(i)}: 1 \leq i \leq r_{j}\right\}$ be a basis of $U_{j, \min }(\mathbf{v})$. There are functionals $\varphi_{j}^{(i)} \in V_{j}^{*}$ with the property $\varphi_{j}^{(i)}\left(b_{j}^{(k)}\right)=\delta_{i k}$. Define $\mathbf{a}_{\mathbf{i}}:=\bigotimes_{j=1}^{d} \varphi_{j}^{\left(i_{j}\right)} \in{ }_{a} \bigotimes_{j=1}^{d} V_{j}^{*}$ and $\mathbf{b}_{\mathbf{i}}:=\bigotimes_{j=1}^{d} b_{j}^{\left(i_{j}\right)} \in{ }_{a} \bigotimes_{j=1}^{d} U_{j, \min }(\mathbf{v})$ for $\mathbf{i}=\left(i_{1}, \ldots, i_{d}\right)$ with $1 \leq i_{j} \leq r_{j}$. Any $\mathbf{u} \in{ }_{a} \bigotimes_{j=1}^{d} U_{j, \min }(\mathbf{v})$ is reproduced by

$$
\mathbf{u}=\sum_{\mathbf{i}} \mathbf{a}_{\mathbf{i}}(\mathbf{u}) \mathbf{b}_{\mathbf{i}} \text {. }
$$

We set

$$
\mathbf{u}_{\mathbf{v}}:=\sum_{\mathbf{i}} \mathbf{a}_{\mathbf{i}}(\mathbf{v}) \mathbf{b}_{\mathbf{i}} \in{ }_{a} \bigotimes_{j=1}^{d} U_{j, \min }(\mathbf{v})
$$

Thus, the theorem follows, if we prove that $\mathbf{v}=\mathbf{u}_{\mathbf{v}} \in{ }_{a} \bigotimes_{j=1}^{d} U_{j, \min }(\mathbf{v})$. Observe that the norm $\left\|\mathbf{v}-\mathbf{u}_{\mathbf{v}}\right\|_{\mathbf{v}}$ is described by $\boldsymbol{\alpha}\left(\mathbf{v}-\mathbf{u}_{\mathbf{v}}\right)$ with normalised $\boldsymbol{\alpha}=\bigotimes_{j=1}^{d} \alpha_{j} \in{ }_{a} \bigotimes_{j=1}^{d} V_{j}^{*}$. If we can show $\boldsymbol{\alpha}\left(\mathbf{v}-\mathbf{u}_{\mathbf{v}}\right)=0$ for all $\boldsymbol{\alpha}$, the norm $\left\|\mathbf{v}-\mathbf{u}_{\mathbf{v}}\right\|_{\vee}$ vanishes and $\mathbf{v}=\mathbf{u}_{\mathbf{v}}$ follows. Thus we need to show the following.

Claim. $\boldsymbol{\alpha}\left(\mathbf{v}-\mathbf{u}_{\mathbf{v}}\right)=0$ holds for all $\boldsymbol{\alpha}=\bigotimes_{j=1}^{d} \alpha_{j} \in{ }_{a} \bigotimes_{j=1}^{d} V_{j}^{*}$.

To prove the claim, split each $\alpha_{j}$ into $\alpha_{j}^{(0)}+\sum_{i} c_{i} \varphi_{j}^{(i)}$ with $c_{i}:=\alpha_{j}\left(b_{j}^{(i)}\right)$ and $\alpha_{j}^{(0)}:=\alpha_{j}-\sum_{i} c_{i} \varphi_{j}^{(i)}$. It follows that $\alpha_{j}^{(0)}\left(b_{j}^{(i)}\right)=0$ for all $i$, i.e.,

$$
\alpha_{j}^{(0)}(u)=0 \quad \text { for all } u \in U_{j, \min }(\mathbf{v})
$$

We expand the product

$$
\boldsymbol{\alpha}=\bigotimes_{j=1}^{d} \alpha_{j}=\bigotimes_{j=1}^{d}\left(\alpha_{j}^{(0)}+\sum_{i} c_{i} \varphi_{j}^{(i)}\right)=\bigotimes_{j=1}^{d}\left(\sum_{i} c_{i} \varphi_{j}^{(i)}\right)+A,
$$

where all products contained in $A$ have at least one factor $\alpha_{j}^{(0)}$. Consider such a product in $A$, where, without loss of generality, we assume $j=1$, i.e., $\alpha_{1}^{(0)} \otimes \boldsymbol{\gamma}_{[1]}$ with $\boldsymbol{\gamma}_{[1]} \in \mathbf{V}_{[1]}$. We conclude that $\left(\alpha_{1}^{(0)} \otimes \boldsymbol{\gamma}_{[1]}\right)\left(\mathbf{u}_{\mathbf{v}}\right)=0$, since $\left(\mathbf{i d}_{[1]} \otimes \alpha_{1}^{(0)}\right)\left(\mathbf{u}_{\mathbf{v}}\right)=\mathbf{0}$ and $\alpha_{1}^{(0)} \otimes \gamma_{[1]}=\gamma_{[1]} \circ\left(\mathbf{i d}_{[1]} \otimes \alpha_{1}^{(0)}\right)$. Furthermore,

$$
\left(\alpha_{1}^{(0)} \otimes \gamma_{[1]}\right)(\mathbf{v})=\alpha_{1}^{(0)}(w) \quad \text { for } w:=\left(i d_{1} \otimes \gamma_{[1]}\right)(\mathbf{v}) .
$$

By definition of $U_{1, \min }(\mathbf{v}), w \in U_{1, \min }(\mathbf{v})$ holds and $\alpha_{1}^{(0)}(w)=\left(\alpha_{1}^{(0)} \otimes \gamma_{[1]}\right)(\mathbf{v})=0$ follows from (4.11). Together, $\left(\alpha_{1}^{(0)} \otimes \gamma_{[1]}\right)\left(\mathbf{v}-\mathbf{u}_{\mathbf{v}}\right)=0$ holds.

It remains to analyse $\left(\bigotimes_{j=1}^{d}\left(\sum_{i} c_{i} \varphi_{j}^{(i)}\right)\right)\left(\mathbf{v}-\mathbf{u}_{\mathbf{v}}\right)=\left(\sum_{\mathbf{i}} \mathbf{c}_{\mathbf{i}} \mathbf{a}_{\mathbf{i}}\right)\left(\mathbf{v}-\mathbf{u}_{\mathbf{v}}\right)$ for $\mathbf{c}_{\mathbf{i}}:=\prod_{j=1}^{d} c_{i_{j}}$. Application to $\mathbf{u}_{\mathbf{v}}$ yields

$$
\left(\sum_{\mathbf{i}} \mathbf{c}_{\mathbf{i}} \mathbf{a}_{\mathbf{i}}\right)\left(\mathbf{u}_{\mathbf{v}}\right)=\sum_{\mathbf{i}} \mathbf{c}_{\mathbf{i}} \mathbf{a}_{\mathbf{i}}(\mathbf{v}) \in \mathbb{R}
$$

(cf. (4.10)). Since this value coincides with $\left(\sum_{\mathbf{i}} \mathbf{c}_{\mathbf{i}} \mathbf{a}_{\mathbf{i}}\right)(\mathbf{v})=\sum_{\mathbf{i}} \mathbf{c}_{\mathbf{i}} \mathbf{a}_{\mathbf{i}}(\mathbf{v})$, we have proved

$$
\left(\bigotimes_{j=1}^{d}\left(\sum_{i} c_{i} \varphi_{j}^{(i)}\right)\right)\left(\mathbf{v}-\mathbf{u}_{\mathbf{v}}\right)=0 \text {. }
$$

Thus the claim follows and thereby $\mathbf{v}=\mathbf{u}_{\mathbf{v}} \in{ }_{a} \bigotimes_{j=1}^{d} U_{j, \min }(\mathbf{v})$. 
4.3.4 $\operatorname{dim}\left(U_{j, \min }(\mathbf{v})\right)=\infty$

For the next theorem we need a further assumption on the norm $\|\cdot\|$. A sufficient condition is that $\|\cdot\|$ is a uniform crossnorm, i.e., it is a crossnorm (cf. (4.2)) and satisfies

$$
\left\|\left(\bigotimes_{j=1}^{d} A_{j}\right)(\mathbf{v})\right\| \leq\left(\prod_{j=1}^{d}\left\|A_{j}\right\|_{V_{j} \leftarrow V_{j}}\right)\|\mathbf{v}\|
$$

for all $A_{j} \in \mathcal{L}\left(V_{j}, V_{j}\right)(1 \leq j \leq d)$ and all $\mathbf{v} \in{ }_{a} \bigotimes_{j=1}^{d} V_{j}$. The uniform crossnorm property implies that $\|\cdot\|$ is a reasonable crossnorm (cf. [21]). Hence, condition (4.6) is ensured (cf. Proposition 4.7a). A further consequence will be needed.

Lemma 4.15 Let $\|\cdot\|$ be a uniform crossnorm on $\mathbf{V}$. Note that $\mathbf{V}=\mathbf{V}_{[d]} \otimes_{a} V_{d}$, where $\mathbf{V}_{[d]}:={ }_{a} \bigotimes_{j=1}^{d-1} V_{j}$.

(a) The map defined by

$$
\|\mathbf{x}\|_{[d]}:=\left\|\mathbf{x} \otimes v_{d}\right\|, \quad \text { where } v_{d} \in V_{d},\left\|v_{d}\right\|_{d}=1,
$$

does not depend on the choice of $v_{d}$. Therefore, it defines a norm on $\mathbf{V}_{[d]}$.

(b) The norm $\|\cdot\|$ is a reasonable crossnorm on $\mathbf{V}_{[d]} \otimes V_{d}$, i.e.,

$$
\left\|\mathbf{x} \otimes v_{d}\right\|=\|\mathbf{x}\|_{[d]}\left\|v_{d}\right\|_{d} \quad \text { and } \quad\left\|\varphi_{[d]} \otimes \varphi_{d}\right\|^{*}=\left\|\varphi_{[d]}\right\|_{[d]}^{*}\left\|\varphi_{d}\right\|_{d}^{*}
$$

for $\mathbf{x} \in \mathbf{V}_{[d]}, v_{d} \in V_{d}, \varphi_{[d]} \in \mathbf{V}_{[d]}^{*}$, and $\varphi_{d} \in V_{d}^{*}$.

(c) For $\varphi_{d} \in V_{d}^{*}$ and $\varphi_{[d]} \in \mathbf{V}_{[d]}^{*}$ the following estimates hold:

$$
\left\|\left(\mathbf{i d}_{[d]} \otimes \varphi_{d}\right)(\mathbf{v})\right\|_{[d]} \leq\left\|\varphi_{d}\right\|_{d}^{*}\|\mathbf{v}\| \quad \text { and } \quad\left\|\left(i d_{d} \otimes \varphi_{[d]}\right)(\mathbf{v})\right\|_{d} \leq\left\|\varphi_{[d]}\right\|_{[d]}^{*}\|\mathbf{v}\| .
$$

Proof. a) Let $\varphi_{d} \in V_{d}^{*}$ be the functional with $\left\|\varphi_{d}\right\|_{d}^{*}=1$ and $\varphi_{d}\left(v_{d}\right)=\left\|v_{d}\right\|_{d}$ (cf. (2.2)). Choose any $w_{d} \in V_{d}$ with $\left\|w_{d}\right\|_{d}=1$ and set $A_{d}:=w_{d} \varphi_{d} \in \mathcal{L}\left(V_{d}, V_{d}\right)$, i.e., $A_{d} v=\varphi_{d}(v) w_{d}$. Because of $\left\|A_{d}\right\|_{V_{d} \leftarrow V_{d}}=$ $\left\|\varphi_{d}\right\|_{d}^{*}\left\|w_{d}\right\|_{d}=1$, the uniform crossnorm property (4.12) with $A_{j}=i d$ for $1 \leq j \leq d-1$ implies $\left\|\mathbf{x} \otimes w_{d}\right\|=$ $\left\|\left(\mathbf{i d}_{[d]} \otimes A_{d}\right)\left(\mathbf{x} \otimes v_{d}\right)\right\| \leq\left\|\mathbf{x} \otimes v_{d}\right\|$. Interchanging the roles of $w_{d}$ and $v_{d}$, we obtain $\left\|\mathbf{x} \otimes v_{d}\right\|=\left\|\mathbf{x} \otimes w_{d}\right\|$.

b1) $\left\|\mathbf{x} \otimes v_{d}\right\|=\|\mathbf{x}\|_{[d]}\left\|v_{d}\right\|_{d}$ in (4.13) follows from the definition of $\|\cdot\|_{[d]}$.

b2) For any elementary tensor $\mathbf{v}=\mathbf{x}_{[d]} \otimes v_{d} \neq 0$ we have $\frac{\left|\left(\varphi_{[d]} \otimes \varphi_{d}\right)(\mathbf{v})\right|}{\|\mathbf{v}\|} \leq\left\|\boldsymbol{\varphi}_{[d]}\right\|_{[d]}^{*}\left\|\varphi_{d}\right\|_{d}^{*}$. Taking the supremum over all $\mathbf{v}=\mathbf{x}_{[d]} \otimes v_{d}$, we obtain

$$
\left\|\boldsymbol{\varphi}_{[d]} \otimes \varphi_{d}\right\|^{*}=\sup _{\mathbf{v} \neq 0} \frac{\left|\left(\boldsymbol{\varphi}_{[d]} \otimes \varphi_{d}\right)(\mathbf{v})\right|}{\|\mathbf{v}\|} \geq \sup _{\mathbf{v}=\mathbf{x}_{[d]} \otimes v_{d} \neq 0} \frac{\left|\left(\boldsymbol{\varphi}_{[d]} \otimes \varphi_{d}\right)(\mathbf{v})\right|}{\|\mathbf{v}\|}=\left\|\boldsymbol{\varphi}_{[d]}\right\|_{[d]}^{*}\left\|\varphi_{d}\right\|_{d}^{*} .
$$

Define $\mathbf{A}:=\bigotimes_{j=1}^{d} A_{j} \in \mathcal{L}(\mathbf{V}, \mathbf{V})$ by $A_{j}=i d(1 \leq j \leq d-1)$ and $A_{d}=\hat{v}_{d} \varphi_{d}$ with $0 \neq \hat{v}_{d} \in V_{d}$. Then $\mathbf{A v}$ is an elementary vector of the form $\mathbf{x}_{[d]} \otimes \hat{v}_{d}$ and $\left\|A_{d}\right\|_{V_{d} \leftarrow V_{d}}=\left\|\hat{v}_{d}\right\|_{d}\left\|\varphi_{d}\right\|_{d}^{*}$ holds. This fact and the crossnorm property $\|\mathbf{A v}\| \leq\left\|\hat{v}_{d}\right\|_{d}\left\|\varphi_{d}\right\|_{d}^{*}\|\mathbf{v}\|$ lead us to

$$
\left\|\varphi_{[d]}\right\|_{[d]}^{*}\left\|\varphi_{d}\right\|_{d}^{*} \geq \frac{\left|\left(\varphi_{[d]} \otimes \varphi_{d}\right)(\mathbf{A v})\right|}{\|\mathbf{A v}\|} \geq \frac{\left|\left(\varphi_{[d]} \otimes \varphi_{d}\right)(\mathbf{A v})\right|}{\left\|\hat{v}_{d}\right\|_{d}\left\|\varphi_{d}\right\|_{d}^{*}\|\mathbf{v}\|}
$$

Since $\left(\boldsymbol{\varphi}_{[d]} \otimes \varphi_{d}\right)(\mathbf{A v})=\left(\varphi_{[d]} \otimes\left(\varphi_{d} A_{d}\right)\right)(\mathbf{v})=\varphi_{d}\left(\hat{v}_{d}\right) \cdot\left(\varphi_{[d]} \otimes \varphi_{d}\right)(\mathbf{v})$, the estimate can be continued by

$$
\left\|\varphi_{[d]}\right\|_{[d]}^{*}\left\|\varphi_{d}\right\|_{d}^{*} \geq \frac{\left|\varphi_{d}\left(\hat{v}_{d}\right)\right|}{\left\|\hat{v}_{d}\right\|_{d}\left\|\varphi_{d}\right\|_{d}^{*}} \frac{\left|\left(\varphi_{[d]} \otimes \varphi_{d}\right)(\mathbf{v})\right|}{\|\mathbf{v}\|} \quad \text { for all } 0 \neq \hat{v}_{d} \in V_{d} .
$$

As $\sup _{\hat{v}_{d} \neq 0} \frac{\left|\varphi_{d}\left(\hat{v}_{d}\right)\right|}{\left\|\hat{v_{d}}\right\|_{d}}=\left\|\varphi_{d}\right\|_{d}^{*}$, it follows that $\frac{\left|\left(\varphi_{[d]} \otimes \varphi_{d}\right)(\mathbf{v})\right|}{\|\mathbf{v}\|} \leq\left\|\boldsymbol{\varphi}_{[d]}\right\|_{[d]}^{*}\left\|\varphi_{d}\right\|_{d}^{*}$ for all $\mathbf{v} \in \mathbf{V}$, so that $\| \boldsymbol{\varphi}_{[d]} \otimes$ $\varphi_{d}\left\|^{*} \leq\right\| \varphi_{[d]}\left\|_{[d]}^{*}\right\| \varphi_{d} \|_{d}^{*}$. Together with the opposite inequality from above, we have proved the second equation in (4.13). 
c) Any $\psi_{[d]} \in \mathbf{V}_{[d]}^{*}$ satisfies $\psi_{[d]} \otimes \varphi_{d}=\psi_{[d]}\left(\mathbf{i d}_{[d]} \otimes \varphi_{d}\right)$. For $\mathbf{v}_{[d]}:=\left(\mathbf{i d}_{[d]} \otimes \varphi_{d}\right)(\mathbf{v})$ there is a $\psi_{[d]} \in \mathbf{V}_{[d]}^{*}$ with $\left\|\psi_{[d]}\right\|_{[d]}^{*}=1$ and $\left|\psi_{[d]}\left(\mathbf{v}_{[d]}\right)\right|=\left\|\mathbf{v}_{[d]}\right\|_{[d]}($ cf. (2.2)). Hence,

$$
\begin{aligned}
\left\|\left(\mathbf{i d}_{[d]} \otimes \varphi_{d}\right)(\mathbf{v})\right\|_{[d]} & =\left|\psi_{[d]}\left(\left(\mathbf{i d}_{[d]} \otimes \varphi_{d}\right)(\mathbf{v})\right)\right|=\left|\left(\psi_{[d]} \otimes \varphi_{d}\right)(\mathbf{v})\right| \\
& \leq\left\|\psi_{[d]} \otimes \varphi_{d}\right\|^{*}\|\mathbf{v}\|=\left\|\psi_{[d]}\right\|_{[d]}^{*}\left\|\varphi_{d}\right\|_{d}^{*}\|\mathbf{v}\|=\left\|\varphi_{d}\right\|_{d}^{*}\|\mathbf{v}\|
\end{aligned}
$$

proves the first inequality in (4.14). The second one can be proved analogously.

Theorem 4.16 Assume that $\mathbf{V}_{\|\cdot\|}$ is a Banach tensor space with a uniform crossnorm $\|\cdot\|$. If $\mathbf{v} \in \mathbf{V}_{\|\cdot\|}$ is the limit of $\mathbf{v}_{n}=\sum_{i=1}^{n} \bigotimes_{j=1}^{d} v_{i, n}^{(j)}$ with the rate

$$
\left\|\mathbf{v}_{n}-\mathbf{v}\right\| \leq o\left(n^{-3 / 2}\right),
$$

then there exists a sequence $\left\{\mathbf{u}_{n}\right\}_{n \in \mathbb{N}} \subset{ }_{a} \bigotimes_{j=1}^{d} U_{j, \min }(\mathbf{v})$ such that $\mathbf{v}=\lim \mathbf{u}_{n}$, i.e., $\mathbf{v} \in \mathbf{U}_{\|\cdot\|}(\mathbf{v})$.

Proof. Use the setting $\mathbf{V} \cong \mathbf{V}_{[d]} \otimes_{a} V_{d}$ from Lemma 4.15. Thus, each $\mathbf{v}_{n} \in \mathbf{V}$ has a representation in $U_{[d], \min }\left(\mathbf{v}_{n}\right) \otimes U_{d, \min }\left(\mathbf{v}_{n}\right)$ with $r:=\operatorname{dim} U_{[d], \min }\left(\mathbf{v}_{n}\right)=\operatorname{dim} U_{d, \min }\left(\mathbf{v}_{n}\right) \leq n$. Renaming $r$ by $n$, we obtain the representation $\mathbf{v}_{n}=\sum_{i=1}^{n} \mathbf{v}_{[d]}^{(i)} \otimes v_{d}^{(i)}$. According to Corollary 3.13b, we can fix a any basis $\left\{v_{d}^{(i)}\right\}$ of $U_{d, \min }\left(\mathbf{v}_{n}\right)$ and recover $\mathbf{v}_{n}=\sum_{i=1}^{n}\left(\mathbf{i d}_{[d]} \otimes \psi_{d}^{(i)}\right)\left(\mathbf{v}_{n}\right) \otimes v_{d}^{(i)}$ from the dual basis $\left\{\psi_{d}^{(i)}\right\}$. We choose $v_{d}^{(i)}$ and $\psi_{d}^{(i)}$ according to Lemma 2.2 with $\left\|v_{d}^{(i)}\right\|_{d}=\left\|\psi_{d}^{(i)}\right\|_{d}^{*}=1$. Define

$$
\mathbf{u}_{n}^{I}:=\sum_{i=1}^{n}\left(\overline{\mathbf{i d}_{[d]} \otimes \psi_{d}^{(i)}}\right)(\mathbf{v}) \otimes v_{d}^{(i)} \in U_{[d], \min }(\mathbf{v}) \otimes_{a} V_{d}
$$

The triangle inequality yields

$$
\begin{aligned}
& \left\|\mathbf{u}_{n}^{I}-\mathbf{v}_{n}\right\|=\left\|\sum_{i=1}^{n}\left(\left(\overline{\mathbf{i d}_{[d]} \otimes \psi_{d}^{(i)}}\right)(\mathbf{v})-\left(\mathbf{i d}_{[d]} \otimes \psi_{d}^{(i)}\right)\left(\mathbf{v}_{n}\right)\right) \otimes v_{d}^{(i)}\right\| \\
& \left.=\left\|\sum_{i=1}^{n}\left(\overline{\mathbf{i d}_{[d]} \otimes \psi_{d}^{(i)}}\right)\left(\mathbf{v}-\mathbf{v}_{n}\right) \otimes v_{d}^{(i)}\right\| \leq \sum_{i=1}^{n} \| \overline{\left(\mathbf{i d}_{[d]} \otimes \psi_{d}^{(i)}\right.}\right)\left(\mathbf{v}-\mathbf{v}_{n}\right) \otimes v_{d}^{(i)} \| \\
& \underset{(4.13)}{=} \sum_{i=1}^{n}\left\|\left(\overline{\mathbf{i d}_{[d]} \otimes \psi_{d}^{(i)}}\right)\left(\mathbf{v}-\mathbf{v}_{n}\right)\right\|_{[d]} \underbrace{\left\|v_{d}^{(i)}\right\|_{d}}_{=1} \\
& \underset{(4.14)}{\leq} \sum_{i=1}^{n} \underbrace{\left\|\psi_{d}^{(i)}\right\|_{d}^{*}}_{=1}\left\|\mathbf{v}-\mathbf{v}_{n}\right\|=n\left\|\mathbf{v}-\mathbf{v}_{n}\right\| .
\end{aligned}
$$

Note that

$$
\mathbf{u}_{n}^{I} \in U_{[d], n} \otimes V_{d} \text { with } U_{[d], n}:=\operatorname{span}\left\{\left(\overline{\operatorname{id}_{[d]} \otimes \psi_{d}^{(i)}}\right)(\mathbf{v}): 1 \leq i \leq n\right\} \subset U_{[d], \min }(\mathbf{v}),
$$

where $\operatorname{dim} U_{[d], n} \leq n$.

On the other side, according to Lemma 2.2, we can choose a basis $\left\{\mathbf{v}_{[d]}^{(i)}\right\}_{i=1}^{n}$ of $U_{[d], \min }\left(\mathbf{v}_{n}\right)$ and its corresponding dual basis $\left\{\chi_{[d]}^{(i)}\right\}_{i=1}^{n}$. An analogous proof shows that

$$
\mathbf{u}_{n}^{I I}:=\sum_{i=1}^{n} \mathbf{v}_{[d]}^{(i)} \otimes\left(\overline{i d_{d} \otimes \boldsymbol{\chi}_{[d]}^{(i)}}\right)(\mathbf{v})
$$

satisfies the properties

$$
\left\|\mathbf{u}_{n}^{I I}-\mathbf{v}_{n}\right\| \leq n\left\|\mathbf{v}-\mathbf{v}_{n}\right\|
$$

and $\mathbf{u}_{n}^{I I} \in \mathbf{V}_{[d]} \otimes_{a} U_{d, n}$, where $U_{d, n}:=\operatorname{span}\left\{\left(\overline{\left(i d_{d} \otimes \boldsymbol{\chi}_{[d]}^{(i)}\right.}\right)(\mathbf{v}): 1 \leq i \leq n\right\}$ has $\operatorname{dim} U_{d, n} \leq n$ and is a subspace of $U_{d, \min }(\mathbf{v})$. 
From Theorem 2.3 we choose the projection $\Phi_{d}$ onto the subspace $U_{d, n}$ and define

$$
\mathbf{u}_{n}:=\left(\mathbf{i d}_{[d]} \otimes \Phi_{d}\right) \mathbf{u}_{n}^{I} \in U_{[d], n} \otimes_{a} U_{d, n} \subset U_{[d], \min }(\mathbf{v}) \otimes_{a} U_{d, \min }(\mathbf{v}) \underset{(3.9)}{\subset}{ }_{a} \bigotimes_{j=1}^{d} U_{j, \min }(\mathbf{v}) .
$$

The uniform crossnorm property (4.12) with $A_{j}=i d(1 \leq j \leq d-1)$ and $A_{d}=\Phi_{d}$ implies the estimate $\left\|\mathbf{i d}_{[d]} \otimes \Phi_{d}\right\|_{\mathbf{V} \leftarrow \mathbf{V}}=\left\|\Phi_{d}\right\|_{\mathbf{V}_{d} \leftarrow \mathbf{V}_{d}} \leq \sqrt{n}$, where the latter bound is given by Theorem 2.3. Therefore,

$$
\begin{gathered}
\left\|\left(\mathbf{i d}_{[d]} \otimes \Phi_{d}\right) \mathbf{v}_{n}-\mathbf{u}_{n}^{I I}\right\|=\left\|\left(\mathbf{i d}_{[d]} \otimes \Phi_{d}\right)\left(\mathbf{v}_{n}-\mathbf{u}_{n}^{I I}\right)\right\| \leq \sqrt{n}\left\|\mathbf{v}_{n}-\mathbf{u}_{n}^{I I}\right\| \underset{(4.16)}{\leq} C n^{3 / 2}\left\|\mathbf{v}-\mathbf{v}_{n}\right\|, \\
\left\|\mathbf{u}_{n}-\left(\mathbf{i d}_{[d]} \otimes \Phi_{d}\right) \mathbf{v}_{n}\right\|=\left\|\left(\mathbf{i d}_{[d]} \otimes \Phi_{d}\right)\left(\mathbf{u}_{n}^{I}-\mathbf{v}_{n}\right)\right\| \leq \sqrt{n}\left\|\mathbf{u}_{n}^{I}-\mathbf{v}_{n}\right\| \underset{(4.15)}{\leq} C n^{3 / 2}\left\|\mathbf{v}-\mathbf{v}_{n}\right\| .
\end{gathered}
$$

Altogether, we get the estimate

$$
\begin{aligned}
\left\|\mathbf{u}_{n}-\mathbf{v}\right\| & =\left\|\left(\mathbf{u}_{n}-\left(\mathbf{i d}_{[d]} \otimes \Phi_{d}\right) \mathbf{v}_{n}\right)+\left(\left(\mathbf{i d}_{[d]} \otimes \Phi_{d}\right) \mathbf{v}_{n}-\mathbf{u}_{n}^{I I}\right)+\left(\mathbf{u}_{n}^{I I}-\mathbf{v}_{n}\right)+\left(\mathbf{v}_{n}-\mathbf{v}\right)\right\| \\
& \leq\left(2 C n^{3 / 2}+n+1\right)\left\|\mathbf{v}-\mathbf{v}_{n}\right\| .
\end{aligned}
$$

The assumption $\left\|\mathbf{v}-\mathbf{v}_{n}\right\| \leq o\left(n^{-3 / 2}\right)$ implies $\left\|\mathbf{u}_{n}-\mathbf{v}\right\| \rightarrow 0$.

\subsection{Minimal closed subspaces in a Hilbert tensor space}

Let $\langle\cdot, \cdot\rangle_{j}$ be a scalar product defined on $V_{j}(1 \leq j \leq d)$, i.e., $V_{j}$ is a pre-Hilbert space. Then $\mathbf{V}={ }_{a} \bigotimes_{j=1}^{d} V_{j}$ is again a pre-Hilbert space with a scalar product which is defined for elementary tensors $\mathbf{v}=\bigotimes_{j=1}^{d} v^{(j)}$ and $\mathbf{w}=\bigotimes_{j=1}^{d} w^{(j)}$ by

$$
\langle\mathbf{v}, \mathbf{w}\rangle=\left\langle\bigotimes_{j=1}^{d} v^{(j)}, \bigotimes_{j=1}^{d} w^{(j)}\right\rangle:=\prod_{j=1}^{d}\left\langle v^{(j)}, w^{(j)}\right\rangle_{j} \quad \text { for all } v^{(j)}, w^{(j)} \in V_{j} .
$$

This bilinear form has a unique extension $\langle\cdot, \cdot\rangle: \mathbf{V} \times \mathbf{V} \rightarrow \mathbb{R}$. One verifies that $\langle\cdot, \cdot\rangle$ is a scalar product, called the induced scalar product. Let $\mathbf{V}$ be equipped with the norm $\|\cdot\|$ corresponding to the induced scalar product $\langle\cdot, \cdot\rangle$. As usual, the Hilbert tensor space $\mathbf{V}_{\|\cdot\|}={ }_{\|\cdot\|} \bigotimes_{j=1}^{d} V_{j}$ is the completion of $\mathbf{V}$ with respect to $\|\cdot\|$. Since the norm $\|\cdot\|$ is derived via (4.17), it is easy to see that $\|\cdot\|$ is a reasonable and even uniform crossnorm.

We recall that orthogonal projections $P \in \mathcal{L}(V, V)$ ( $V$ Hilbert space) are selfadjoint projections. $P$ is an orthogonal projection onto the closed subspace $U:=\operatorname{range}(P) \subset V$, which leads to the direct sum $V=U \oplus U^{\perp}$, where $U^{\perp}=\operatorname{range}(i d-P)$. Vice versa, each closed subspace $U \subset V$ defines an orthogonal projections $P$ with $U=\operatorname{range}(P)$.

Lemma 4.17 Let $V_{j}$ be Hilbert spaces with closed ${ }^{4}$ subspaces $U_{j} \subset V_{j}$. The norm $\|\cdot\|$ of the Hilbert tensor space $\|\cdot\| \bigotimes_{j=1}^{d} V_{j}$ is defined via the scalar product (4.17). Then

$$
\bigcap_{1 \leq j \leq d}\left(U_{j} \otimes_{\|\cdot\|} V_{[j]}\right)=\| \cdot \bigotimes_{j=1}^{d} U_{j}, \quad \text { where } V_{[j]}:={ }_{a} \bigotimes_{i \neq j} V_{i} .
$$

Proof. We consider the case $d=2$ only ( $d \geq 3$ can be obtained by induction). Then the assertion to be proved is

$$
\left(U_{1} \otimes_{\|\cdot\|} V_{2}\right) \cap\left(V_{1} \otimes_{\|\cdot\|} U_{2}\right)=U_{1} \otimes_{\|\cdot\|} U_{2} .
$$

\footnotetext{
${ }^{4}$ Because of Lemma 4.4, the closedness of the subspaces $U_{j}$ is not required for the statement. The assumption allows to state that $V_{j}=U_{j} \oplus U_{j}^{\perp}$.
} 
The analogous statement for the algebraic tensor spaces holds by Lemma 3.9. The general rule $\overline{X \cap Y} \subset \bar{X} \cap \bar{Y}$ $(\cdot$ is the closure with respect to $\|\cdot\|)$ implies that

$$
\begin{aligned}
U_{1} \otimes_{\|\cdot\|} U_{2} & =\overline{U_{1} \otimes_{a} U_{2}}=\overline{\left(U_{1} \otimes_{a} V_{2}\right) \cap\left(V_{1} \otimes_{a} U_{2}\right)} \\
& \subset \overline{U_{1} \otimes_{a} V_{2}} \cap \overline{V_{1} \otimes_{a} U_{2}}=\left(U_{1} \otimes_{\|\cdot\|} V_{2}\right) \cap\left(V_{1} \otimes_{\|\cdot\|} U_{2}\right) .
\end{aligned}
$$

The lemma is proved, if the opposite inclusion holds:

$$
\left(U_{1} \otimes_{\|\cdot\|} V_{2}\right) \cap\left(V_{1} \otimes_{\|\cdot\|} U_{2}\right) \subset U_{1} \otimes_{\|\cdot\|} U_{2} .
$$

Let $\mathbf{v} \in U_{1} \otimes_{\|\cdot\|} V_{2}$. By definition, there is a sequence $\mathbf{v}_{n} \in U_{1} \otimes_{a} V_{2}$ with $\mathbf{v}_{n} \rightarrow \mathbf{v}$. Let $P_{1}$ be the orthogonal projection onto $U_{1}$. Then $\left(P_{1} \otimes i d_{2}\right) \mathbf{v}_{n}=\mathbf{v}_{n}$ proves $\mathcal{P}_{1} \mathbf{v}=\mathbf{v}$ for the extension $\mathcal{P}_{1}:=\overline{P_{1} \otimes i d_{2}}$. Similarly, $\mathcal{P}_{2} \mathbf{v}=\mathbf{v}$ follows with $\mathcal{P}_{2}:=\overline{i d_{1} \otimes P_{2}}$, where $P_{2}$ is the orthogonal projection onto $U_{2}$. Since $P_{1} \otimes i d_{2}$ and $i d_{1} \otimes P_{2}$ commute, also the product $P_{1} \otimes P_{2}$ is an orthogonal projection. Its range is $U_{1} \otimes_{a} U_{2}$, while $U_{1} \otimes_{\|\cdot\|} U_{2}$ is the range of its extension $\mathcal{P}:=\overline{P_{1} \otimes P_{2}}=\mathcal{P}_{1} \mathcal{P}_{2}=\mathcal{P}_{2} \mathcal{P}_{1}$. Hence, $\mathcal{P}_{1} \mathbf{v}=\mathcal{P}_{2} \mathbf{v}=\mathbf{v}$ implies $\mathcal{P} \mathbf{v}=\mathbf{v}$, i.e., $\mathbf{v} \in U_{1} \otimes_{\|\cdot\|} U_{2}$. This ends the proof of (4.18).

Lemma 4.18 Let $V_{i}(i=1,2)$ be Hilbert spaces, and $U_{1} \subset V_{1}$ a closed subspace. Then the direct sum $V_{1}=U_{1} \oplus U_{1}^{\perp}$ implies

$$
V_{1} \otimes_{\|\cdot\|} V_{2}=\left(U_{1} \otimes_{\|\cdot\|} V_{2}\right) \oplus\left(U_{1}^{\perp} \otimes_{\|\cdot\|} V_{2}\right) .
$$

Proof. Consider the ranges of $\mathcal{P}_{1}=\overline{P_{1} \otimes i d_{2}}$ and id $-\mathcal{P}_{1}$, where $P_{1}$ is the orthogonal projection onto $U_{1}$.

Different from Theorem 4.16 for the Banach tensor space setting, we need no assumption on the speed of the convergence $\mathbf{v}_{n} \rightarrow \mathbf{v}$ to obtain the result $\mathbf{v} \in \mathbf{U}_{\|\cdot\|}(\mathbf{v})$.

Theorem 4.19 Assume that $V_{j}$ are Hilbert spaces and that $\mathbf{V}$ is equipped with the norm $\|\cdot\|$ corresponding to the induced scalar product. Then for all $\mathbf{v} \in \mathbf{V}_{\|\cdot\|}$ it follows that $\mathbf{v} \in \mathbf{U}_{\|\cdot\|}(\mathbf{v})$.

Proof. 1) In order to simplify the notation, we set $U_{j}:={\overline{U_{j, \min }(\mathbf{v})}}^{\|\cdot\|_{j}}$ for $1 \leq j \leq d$. For all $1 \leq j \leq d$ we may write $\mathbf{V}_{\|\cdot\|}$ as $V_{j} \otimes_{\|\cdot\|} V_{[j]}$. If we succeed to prove $\mathbf{v} \in U_{j} \otimes_{\|\cdot\|} V_{[j]}$, Lemma 4.17 implies $\mathbf{v} \in\|\cdot\| \otimes_{j=1}^{d} U_{j}=$ $\mathbf{U}_{\|\cdot\|}(\mathbf{v})$.

2) According to $\mathbf{V}_{\|\cdot\|}=\left(U_{j} \otimes_{\|\cdot\|} \mathbf{V}_{[j]}\right) \oplus\left(U_{j}^{\perp} \otimes_{\|\cdot\|} \mathbf{V}_{[j]}\right)$ from Lemma 4.18, we split $\mathbf{v}$ into

$$
\mathbf{v}=\mathbf{v}_{\|}+\mathbf{v}_{\perp} \quad \text { with } \mathbf{v}_{\|} \in U_{j} \otimes_{\|\cdot\|} \mathbf{V}_{[j]} \text { and } \mathbf{v}_{\perp} \in U_{j}^{\perp} \otimes_{\|\cdot\|} \mathbf{V}_{[j]} .
$$

For an indirect proof we assume $\mathbf{v}_{\perp} \neq 0$. Then there are $v_{j} \in U_{j}^{\perp}$ and $\mathbf{v}_{[j]} \in \mathbf{V}_{[j]}$ with $\left\langle v_{j} \otimes \mathbf{v}_{[j]}, \mathbf{v}_{\perp}\right\rangle=$ $\left\langle v_{j} \otimes \mathbf{v}_{[j]}, \mathbf{v}\right\rangle \neq 0$ (otherwise there are no algebraic tensors converging to $\mathbf{v}_{\perp}$ ). For $\boldsymbol{\varphi}_{[j]}:=\left\langle\mathbf{v}_{[j]}, \cdot\right\rangle_{[j]} \in \mathbf{V}_{[j]}^{*}$ one verifies

$$
\left\langle v_{j} \otimes \mathbf{v}_{[j]}, \mathbf{v}\right\rangle=\left\langle v_{j},\left(i d_{j} \otimes \boldsymbol{\varphi}_{[j]}\right)(\mathbf{v})\right\rangle .
$$

The definition of $U_{j, \min }(\mathbf{v})$ yields $\left(i d_{j} \otimes \varphi_{[j]}\right)(\mathbf{v}) \in U_{j}$. Since $v_{j} \in U_{j}^{\perp}$, we obtain the contradiction $\left\langle v_{j} \otimes \mathbf{v}_{[j]}, \mathbf{v}_{\perp}\right\rangle=\left\langle v_{j} \otimes \mathbf{v}_{[j]}, \mathbf{v}\right\rangle=0$. Hence $\mathbf{v}_{\perp}=0$ proves the statement $\mathbf{v} \in U_{j} \otimes_{\|\cdot\|} V_{[j]}$ needed in part 1).

So far, we have assumed that the norm $\|\cdot\|$ of the Hilbert space $\mathbf{V}$ corresponds to the induced scalar product. In principle, we may also define another scalar product $\langle\cdot, \cdot\rangle_{\mathbf{V}}$ on $\mathbf{V}$ together with another norm $\|\cdot\|_{\mathbf{V}}$. In this case, we have to assume that $\|\cdot\|_{\mathbf{V}}$ is a uniform crossnorm (at least, $\left\|\left(\bigotimes_{j=1}^{d} A_{j}\right)(\mathbf{v})\right\| \leq$ $C\left(\prod_{j=1}^{d}\left\|A_{j}\right\|_{V_{j} \leftarrow V_{j}}\right)\|\mathbf{v}\|$ must hold for some constant $C$ ). This ensures that the projections $\mathcal{P}_{j}$ (as defined in the proof of Lemma 4.17) belong to $\mathcal{L}(\mathbf{V}, \mathbf{V})$. Furthermore, (4.6) holds. Scalar products like $\left\langle v_{j} \otimes \mathbf{v}_{[j]}, \mathbf{v}\right\rangle$ in the proof above are to be replaced $\left(\varphi_{j} \otimes \varphi_{[j]}\right)(\mathbf{v})$, where, as usual, $\varphi_{j} \in V_{j}^{*}$ is defined via $\varphi_{j}(\cdot)=\left\langle v_{j}, \cdot\right\rangle_{j}$. Then we can state again that $\mathbf{v} \in \mathbf{U}_{\|\cdot\|}(\mathbf{v})$. 


\section{On the best $\mathcal{T}_{\mathrm{r}}$ approximation in a Banach tensor space}

\subsection{Main statement}

Theorem 5.1 Let $\mathbf{V}_{\|\cdot\|}$ be a reflexive Banach tensor space with a norm satisfying (4.6). Then for each $\mathbf{v} \in \mathbf{V}_{\|\cdot\|}$ there exists $\mathbf{w} \in \mathcal{T}_{\mathbf{r}}$ such that

$$
\|\mathbf{v}-\mathbf{w}\|=\min _{\mathbf{u} \in \mathcal{T}_{\mathbf{r}}}\|\mathbf{v}-\mathbf{u}\|
$$

Proof. Combine Theorem 2.8 and Proposition 5.2 below.

Proposition 5.2 Let $\mathbf{V}_{\|\cdot\|}$ be a Banach tensor space with a norm with a norm satisfying (4.6). Then the set $\mathcal{T}_{\mathbf{r}}$ is weakly closed.

Proof. Let $\left\{\mathbf{v}_{n}\right\} \subset \mathcal{T}_{\mathbf{r}}$ be such that $\mathbf{v}_{n} \rightarrow \mathbf{v}$. Then there are subspaces $U_{j, n} \subset V_{j}$ such that $\mathbf{v}_{n} \in U_{j, n}$ with $\operatorname{dim} U_{j, n}=r_{j}$. Since $U_{j, \min }\left(\mathbf{v}_{n}\right) \subset U_{j, n}, \operatorname{dim} U_{j, \min }\left(\mathbf{v}_{n}\right) \leq r_{j}$ holds for all $n \in \mathbb{N}$. In consequence, by Theorem 4.13, $\operatorname{dim} U_{j, \min }(\mathbf{v}) \leq r_{j}$. Thus, $U_{j, \min }(\mathbf{v})$ is finite dimensional. From Theorem 4.14 we conclude that $\mathbf{v} \in{ }_{a} \bigotimes_{j=1}^{d} U_{j, \min }(\mathbf{v})$ and, thereby, $\mathbf{v} \in \mathcal{T}_{\mathbf{r}}$.

Corollary 5.3 A statement analogous to Theorem 5.1 also holds for the set $\mathcal{H}_{\mathfrak{r}}$ appearing for the hierarchical format from [12] and the format from [20]. The proof uses the fact that $\mathcal{H}_{\mathfrak{r}}$ is weakly closed.

\subsection{Generalisation to the intersection of finitely many Banach tensor spaces}

We recall that the assumption (4.6) implies ${ }_{a} \bigotimes_{j=1}^{d} V_{j}^{*} \subset\left({ }_{a} \bigotimes_{j=1}^{d} V_{j}\right)^{*}$ (cf. Proposition 4.7b). For certain Banach tensor spaces this property does not hold. Therefore, we have to check whether some of the results given in the previous section can be extended to this case. Thus, in this section we introduce the so-called Intersection Tensor Spaces. Also we study the sequences of minimal subspaces in this framework in order to prove the existence of the best $\mathcal{T}_{\mathbf{r}}$ approximation. To illustrate this situation we give the following example.

Recall that $\|f\|_{C^{1}(I)}=\max _{x \in I}\left\{|f(x)|,\left|f^{\prime}(x)\right|\right\}$ is the norm of continuously differentiable functions in one variable $x \in I \subset \mathbb{R}$. The naming $\|\cdot\|_{1, \text { mix }}$ of the following norm is derived from the mixed derivative involved.

Example 5.4 Let $I$ and $J$ be compact intervals in $\mathbb{R}$ and consider $V=\left(C^{1}(I),\|\cdot\|_{C^{1}(I)}\right)$ and $W=\left(C^{1}(J),\|\cdot\|_{C^{1}(J)}\right)$. For the tensor space $V \otimes_{a} W$ we introduce the norm

$$
\|\varphi\|_{C_{\text {mix }}^{1}(I \times J)}:=\|\varphi\|_{1, \text { mix }}:=\max _{(x, y) \in I \times J}\left\{|\varphi(x, y)|,\left|\frac{\partial}{\partial x} \varphi(x, y)\right|,\left|\frac{\partial}{\partial y} \varphi(x, y)\right|,\left|\frac{\partial^{2}}{\partial x \partial y} \varphi(x, y)\right|\right\} .
$$

It can be shown that $\|\cdot\|_{1, \operatorname{mix}}$ is a reasonable crossnorm. However, the standard norm of $C^{1}(I \times J)$ given by

$$
\|\varphi\|_{C^{1}(I \times J)}:=\max _{(x, y) \in I \times J}\left\{|\varphi(x, y)|,\left|\frac{\partial}{\partial x} \varphi(x, y)\right|,\left|\frac{\partial}{\partial y} \varphi(x, y)\right|\right\}
$$

is no reasonable crossnorm.

We have seen that the space $C^{1}(I \times J)$ is not the straightforward result of the tensor product $C^{1}(I) \otimes$ $C^{1}(J)$. The norm $\|\cdot\|_{1, \text { mix }}$ from (5.1) turns out to be a reasonable crossnorm, but then the resulting space $C_{\text {mix }}^{1}(I \times J)$ is a smaller space than $C^{1}(I \times J)$. Vice versa, the dual norm $\|\cdot\|_{C^{1}(I \times J)}^{*}$ of $C^{1}(I \times J)$ is not bounded for $v^{*} \otimes w^{*} \in V^{*} \otimes W^{*}$. Therefore, it is not a reasonable crossnorm.

The family of Sobolev spaces $H^{m, p}\left(I_{j}\right)$ for $m=0,1, \ldots, N$ is an example for a scale of Banach spaces which we introduce below. From now on, we fix integers $N_{j}$ and denote the $j$-th scale by

$$
V_{j}=V_{j}^{(0)} \supset V_{j}^{(1)} \supset \ldots \supset V_{j}^{\left(N_{j}\right)} \text { with dense embedding, }
$$


which means that $V_{j}^{(n)}$ is a dense subspace of $\left(V_{j}^{(n-1)},\|\cdot\|_{j, n-1}\right)$ for $n=1, \ldots, N_{j}$. This fact implies that the corresponding norms satisfy

$$
\|\cdot\|_{j, n} \gtrsim\|\cdot\|_{j, m} \text { for } N_{j} \geq n \geq m \geq 0 \text { on } V_{j}^{(n)} .
$$

Thus, without loss of generality, we assume that (after suitable scaling) $\|\cdot\|_{j, n} \geq\|\cdot\|_{j, m}$ holds for $N_{j} \geq n \geq m \geq 0$ and $1 \leq j \leq d$.

Lemma 5.5 Under the given assumptions for (5.3), all $V_{j}^{(n)}\left(1 \leq n \leq N_{j}\right)$ are dense in $\left(V_{j}^{(0)},\|\cdot\|_{j, 0}\right)$.

Proof. Given $\varepsilon>0$ and $v^{(0)} \in V_{j}^{(0)}$, the dense embeddings imply that we can choose $v^{(1)} \in V_{j}^{(1)}$, $v^{(2)} \in V_{j}^{(2)}, \ldots$ with $\left\|v^{(m-1)}-v^{(m)}\right\|_{j, n-1} \leq \varepsilon / N_{j}$ for $1 \leq m \leq n \leq N_{j}$. The triangle inequality shows $\left\|v^{(0)}-v^{(n)}\right\|_{j, 0} \leq \sum_{m=1}^{n}\left\|v^{(m-1)}-v^{(m)}\right\|_{j, 0} \leq \sum_{m=1}^{n}\left\|v^{(m-1)}-v^{(m)}\right\|_{j, m-1} \leq \frac{n}{N_{j}} \varepsilon \leq \varepsilon$.

Definition 5.6 Under the given assumptions for (5.3) we say that a subset $\mathcal{N} \subset \mathbb{N}_{0}^{d}$ is an admissible index set, if it satisfies

$$
\begin{aligned}
\mathbf{n} & \in \mathcal{N} \text { if and only if } n_{j} \leq N_{j} \text { for } 1 \leq j \leq d, \\
\mathbf{0} & :=(0, \ldots, 0) \in \mathcal{N} \text { and } \\
\mathbf{N}_{j} & :=(\underbrace{0, \ldots, 0}_{j-1}, N_{j}, \underbrace{0, \ldots, 0}_{d-j}) \in \mathcal{N} \text { for } 1 \leq j \leq d .
\end{aligned}
$$

For each $\mathbf{n}$ in an admissible index set $\mathcal{N}$, we define the tensor space

$$
\mathbf{V}^{(\mathbf{n})}:={ }_{a} \bigotimes_{j=1}^{d} V_{j}^{\left(n_{j}\right)}
$$

All spaces $\mathbf{V}^{(\mathbf{n})}$ are subspaces of $\mathbf{V}^{(\mathbf{0})}={ }_{a} \bigotimes_{j=1}^{d} V_{j}$ (recall that $V_{j}=V_{j}^{(0)}$ ). Assume that the following conditions hold:

(a) For each admissible $\mathbf{n} \in \mathbb{N}_{0}^{d}$, a norm $\|\cdot\|_{\mathbf{n}}$ on $\mathbf{V}^{(\mathbf{n})}$ exists satisfying $\|\cdot\|_{\mathbf{n}} \leq\|\cdot\|_{\mathbf{m}}$ for $\mathbf{n} \leq \mathbf{m} \in \mathcal{N}$ and

(b) the norm $\|\cdot\|_{\mathbf{0}}$ on $\mathbf{V}^{(\mathbf{0})}={ }_{a} \bigotimes_{j=1}^{d} V_{j}$ satisfy (4.6).

Now, we introduce the Banach tensor space

$$
\mathbf{V}_{\|\cdot\|}^{(\mathbf{n})}:=\|\cdot\|_{\mathbf{n}} \bigotimes_{j=1}^{d} V_{j}^{\left(n_{j}\right)}
$$

Note that for each $\mathbf{n} \in \mathcal{N}$, if $\mathbf{n} \leq \mathbf{m} \in \mathcal{N}$, then $\mathbf{V}_{\|\cdot\|}^{(\mathbf{m})} \subset \mathbf{V}_{\|\cdot\|}^{(\mathbf{n})}$. From Lemma 3.14 one derives the following result.

Lemma 5.7 Let $\mathcal{N} \subset \mathbb{N}_{0}^{d}$ be an admissible index set. Then the following statements hold:

(a) $\mathbf{V}^{(\mathbf{n})}=\bigcap_{j=1}^{d} \mathbf{V}^{\left(\mathbf{n}_{j}\right)}$ for all $\mathbf{n} \in \mathcal{N}$, where $\mathbf{n}_{j}:=(\underbrace{0, \ldots, 0}_{j-1}, n_{j}, \underbrace{0, \ldots, 0}_{d-j}) \in \mathbb{N}_{0}^{d}$, for $1 \leq j \leq d$.

(b) $\bigcap_{\mathbf{n} \in \mathcal{N}} \mathbf{V}^{(\mathbf{n})}=\mathbf{V}^{\left(N_{1}, \ldots, N_{d}\right)}={ }_{a} \bigotimes_{j=1}^{d} V_{j}^{\left(N_{j}\right)}$.

Proof. From Lemma 3.14 we have

$$
\bigcap_{j=1}^{d} \mathbf{V}^{\left(\mathbf{n}_{j}\right)}={ }_{a} \bigotimes_{j=1}^{d} \bigcap_{i=1}^{d} V_{j}^{\left(n_{j} \delta_{i, j}\right)}={ }_{a} \bigotimes_{j=1}^{d} V_{j}^{\left(n_{j}\right)}
$$


and statement (a) follows. Also, by Lemma 3.14,

$$
\bigcap_{\mathbf{n} \in \mathcal{N}} \mathbf{V}^{(\mathbf{n})}={ }_{a} \bigotimes_{j=1}^{d} \bigcap_{\mathbf{n} \in \mathcal{N}} V_{j}^{\left(n_{j}\right)}
$$

and, by (5.3), statement (b) is proved.

Let $\mathcal{N} \subset \mathbb{N}_{0}^{d}$ be an admissible index set. From Lemma $5.7 \mathrm{~b}$ it follows that the intersection of the set of tensor spaces $\left\{\mathbf{V}^{(\mathbf{n})}: \mathbf{n} \in \mathcal{N}\right\}$ is the tensor space $\mathbf{V}^{\left(N_{1}, \ldots, N_{d}\right)} \subset \mathbf{V}_{\|\cdot\|}^{\left(N_{1}, \ldots, N_{d}\right)}$. Observe that the index $\left(N_{1}, \ldots, N_{d}\right)$ not necessarily belongs to the index set $\mathcal{N}$. Also, by Lemma 5.7 a, we obtain the following minimal representation

$$
\mathbf{V}^{\left(N_{1}, \ldots, N_{d}\right)}=\bigcap_{j=1}^{d} \mathbf{V}^{\left(\mathbf{N}_{j}\right)}
$$

Next, we introduce the Banach space induced by intersection of the set of Banach tensor spaces $\left\{\mathbf{V}_{\|\cdot\|}^{(\mathbf{n})}: \mathbf{n} \in \mathcal{N}\right\}$.

Definition 5.8 Let $\mathcal{N} \subset \mathbb{N}_{0}^{d}$ be an admissible index set. The Banach space $\mathbf{V}_{\|\cdot\|_{\mathcal{N}}}$ induced by the intersection of the set of Banach tensor spaces $\left\{\mathbf{V}_{\|\cdot\|}^{(\mathbf{n})}: \mathbf{n} \in \mathcal{N}\right\}$ is defined by

$$
\mathbf{V}_{\|\cdot\|_{\mathcal{N}}}:=\bigcap_{\mathbf{n} \in \mathcal{N}} \mathbf{V}_{\|\cdot\|}^{(\mathbf{n})} \text { with the intersection norm }\|\mathbf{v}\|_{\mathcal{N}}:=\max _{\mathbf{n} \in \mathcal{N}}\|\mathbf{v}\|_{\mathbf{n}}
$$

or an equivalent one.

Next, we consider particular tensors from the tensor space $\mathbf{V}^{(\mathbf{0})}$.

Proposition 5.9 Let $\mathcal{N} \subset \mathbb{N}_{0}^{d}$ be an admissible index set. Then

$$
\mathbf{V}^{(\mathbf{0})} \cap \mathbf{V}_{\|\cdot\|_{\mathcal{N}}}=\mathbf{V}^{\left(N_{1}, \ldots, N_{d}\right)}
$$

holds. In particular, each $\mathbf{v} \in \mathbf{V}^{(\mathbf{0})} \cap \mathbf{V}_{\|\cdot\|_{\mathcal{N}}}$ has a representation $\mathbf{v}=\sum_{i=1}^{r} \bigotimes_{j=1}^{d} v_{j}^{(i)}$ with $v_{j}^{(i)} \in V_{j}^{\left(N_{j}\right)}$ and a minimal number $r$ of terms.

Proof. First, observe that $\mathbf{V}^{\left(N_{1}, \ldots, N_{d}\right)} \subset \mathbf{V}^{(\mathbf{0})}$ and $\mathbf{V}^{\left(N_{1}, \ldots, N_{d}\right)} \subset \mathbf{V}_{\|\cdot\|_{\mathcal{N}}}$, thus $\mathbf{V}^{\left(N_{1}, \ldots, N_{d}\right)} \subset \mathbf{V}^{(\mathbf{0})} \cap \mathbf{V}_{\|\cdot\|_{\mathcal{N}}}$. To prove equality, assume that $\mathbf{v} \in \mathbf{V}^{(\mathbf{0})} \cap \mathbf{V}_{\|\cdot\|_{\mathcal{N}}}$. Note that $\mathbf{v} \in \bigcap_{\mathbf{n} \in \mathcal{N}} V_{\|\cdot\|}^{(\mathbf{n})} \subset \mathbf{V}_{\|\cdot\|_{\mathbf{N}_{j}}}^{\left(\mathbf{N}_{j}\right)}$ for $1 \leq j \leq d$. Since $\mathbf{v}$ also belongs to $\mathbf{V}^{(\mathbf{0})}$, we consider for any $j \in\{1, \ldots, d\}$ the matricisation of $\mathbf{v}=\sum_{i=1}^{r} \bigotimes_{j=1}^{d} v_{j}^{(i)}$ yielding $\sum_{i=1}^{r} v_{j}^{(i)} \otimes \mathbf{v}_{[j]}^{i}$ with $v_{j}^{(i)} \in V_{j}^{(0)}$ and $\mathbf{v}_{[j]}^{i} \in{ }_{a} \bigotimes_{k \neq j} V_{k}^{(0)}$. By Lemma 3.8, the vectors $\mathbf{v}_{[j]}^{i}(1 \leq i \leq r)$ are linearly independent. Hence, there are functionals $\varphi_{[j]}^{(k)} \in \mathbf{V}_{[j]}^{*}$ with $\varphi_{[j]}^{(k)}\left(\mathbf{v}_{[j]}^{i}\right)=\delta_{k i}$. Then $\left(i d_{k} \otimes \varphi_{[j]}^{(k)}\right)(\mathbf{v})=$ $v_{j}^{(k)} \in V_{j}^{(0)}$, follows for $1 \leq k \leq r$. The assertion $v_{j}^{(k)} \in V_{j}^{\left(N_{j}\right)}$ is equivalent to $\left|\psi\left(v_{j}^{(k)}\right)\right|<\infty$ for all $\psi \in\left(V_{j}^{\left(N_{j}\right)}\right)^{\prime}$. Then $\psi\left(v_{j}^{(k)}\right)=\psi\left(\left(i d_{j} \otimes \varphi_{[j]}^{(k)}\right)(\mathbf{v})\right)$ is finite, because $\left(i d_{j} \otimes \varphi_{[j]}^{(k)}\right)(\mathbf{v}) \in V_{j}^{(0)}$ and $\psi \in\left(V_{j}^{\left(N_{j}\right)}\right)^{\prime} \subset$ $\left(V_{j}^{(0)}\right)^{\prime}$. Thus we show that $\mathbf{v} \in \mathbf{V}^{\left(\mathbf{N}_{j}\right)}$ for $1 \leq j \leq d$, i.e., $\mathbf{v} \in \bigcap_{j=1}^{d} \mathbf{V}^{\left(\mathbf{N}_{j}\right)}$. Finally, by (5.8), the proposition follows.

Corollary 5.10 The set $\mathbf{V}^{\left(N_{1}, \ldots, N_{d}\right)}$ is dense in $\mathbf{V}_{\|\cdot\|_{\mathcal{N}}}$ with respect to the $\|\cdot\|_{\mathcal{N}^{-}}$topology.

Proof. The inclusions $\mathbf{V}^{\left(N_{1}, \ldots, N_{d}\right)} \subset \mathbf{V}^{(\mathbf{n})} \subset \mathbf{V}_{\|\cdot\|}^{(\mathbf{n})}$ are dense for all $\mathbf{n} \in \mathcal{N}$ (cf. (5.3)). Definition (5.9) yields the assertion.

Example 5.11 Fix $N>0$ and consider the Sobolev spaces $V_{j}^{n}=H^{n, p}\left(I_{j}\right)$ for $0 \leq n \leq N$ and $1 \leq j \leq d$. The standard choice of $\mathcal{N}$ is given by

$$
\left.\mathcal{N}:=\left\{\mathbf{n} \in \mathbb{N}_{0}^{d} \text { with }|\mathbf{n}| \leq N\right\} \quad \text { (here } N_{j}=N \text { for all } 1 \leq j \leq d\right) .
$$


In this situation we have $\mathbf{V}^{\mathbf{n}}=H^{n_{1}, p}\left(I_{1}\right) \otimes_{a} \cdots \otimes_{a} H^{n_{d}, p}\left(I_{d}\right)$ for each $\mathbf{n} \in \mathcal{N}$, and

$$
\mathbf{V}^{(N, \ldots, N)}={ }_{a} \bigotimes_{j=1}^{d} H^{N, p}\left(I_{j}\right)
$$

The choice of the norm in $\mathbf{V}^{\mathbf{n}}$ is

$$
\|f\|_{\mathbf{n}}:=\left(\sum_{\mathbf{0} \leq \mathbf{k} \leq \mathbf{n}} \int_{\mathbf{I}}\left|\partial^{\mathbf{k}} f\right|^{p} \mathrm{~d} x\right)^{1 / p}
$$

while in $\mathbf{V}_{\|\cdot\|_{\mathcal{N}}}$ we take

$$
\|f\|_{\mathcal{N}}:=\left(\sum_{\mathbf{n} \in \mathcal{N}}\|f\|_{\mathbf{n}}^{p}\right)^{1 / p}
$$

which is equivalent to the usual norm $\|\cdot\|_{N, p}$. Then, by Corollary 5.10,

$$
\|\cdot\|_{N, p} \bigotimes_{j=1}^{d} H^{N, p}\left(I_{j}\right)=\bigcap_{\mathbf{n} \in \mathcal{N}} \overline{H^{n_{1}, p}\left(I_{1}\right) \otimes_{a} \cdots \otimes_{a} H^{n_{d}, p}\left(I_{d}\right)}\|\cdot\|_{\mathbf{n}} .
$$

Observe that $\|\cdot\|_{N, p} \bigotimes_{j=1}^{d} H^{N, p}\left(I_{j}\right)$ is a Banach subspace of the Banach space $H^{N, p}(\mathbf{I})$. Moreover, for each $\mathbf{N}_{j}=(0, \ldots, 0, N, 0, \ldots, 0) \in \mathcal{N}$, we have

$$
\|f\|_{\mathbf{N}_{j}}=\left(\sum_{k=0}^{N} \int_{\mathbf{I}}\left|\partial_{x_{j}}^{k} f\right|^{p} \mathrm{~d} x\right)^{1 / p}
$$

which is clearly a crossnorm in

$$
\mathbf{V}^{\left(\mathbf{N}_{j}\right)}=L^{p}\left(I_{1}\right) \otimes_{a} \cdots \otimes_{a} L^{p}\left(I_{j-1}\right) \otimes_{a} H^{N, p}\left(I_{j}\right) \otimes_{a} L^{p}\left(I_{j+1}\right) \otimes \cdots \otimes_{a} L^{p}\left(I_{d}\right),
$$

for $1 \leq j \leq d$. In particular for $p=2$ we obtain

$$
H^{N}(\mathbf{I})=\|\cdot\|_{N, 2} \bigotimes_{j=1}^{d} H^{N}\left(I_{j}\right)=\bigcap_{\mathbf{n} \in \mathcal{N}} \overline{H^{n_{1}}\left(I_{1}\right) \otimes_{a} \cdots \otimes_{a} H^{n_{d}}\left(I_{d}\right)}{ }^{\|\cdot\|_{\mathbf{n}}},
$$

and in this case the norm $\|\cdot\|_{\mathbf{N}_{j}}$ in

$$
\mathbf{V}^{\left(\mathbf{N}_{j}\right)}=L^{2}\left(I_{1}\right) \otimes_{a} \cdots \otimes_{a} L^{2}\left(I_{j-1}\right) \otimes_{a} H^{N}\left(I_{j}\right) \otimes_{a} L^{2}\left(I_{j+1}\right) \otimes \cdots \otimes_{a} L^{2}\left(I_{d}\right)
$$

is generated by the induced scalar product (4.17) for $1 \leq j \leq d$. In consequence, it is a reasonable crossnorm.

Note that Proposition 5.9 states that all functions from the algebraic tensor space ${ }_{a} \bigotimes_{j=1}^{d} C^{0}\left(I_{j}\right) \cap C^{1}(\mathbf{I})$ are already in $\mathbf{V}_{\text {mix }}=C_{\text {mix }}^{1}(\mathbf{I})$ (see Example 5.1), which is a proper subspace of $C^{1}(\mathbf{I})$.

Example 5.12 Fix $N>0$ and consider $V_{j}^{n}=H^{n, p}\left(I_{j}\right)$ for $0 \leq n \leq N$ and $1 \leq j \leq d$. Now, we consider the set

$$
\mathcal{N}:=\left\{\mathbf{n} \in \mathbb{N}_{0}^{d} \text { with }|\mathbf{n}| \leq N\right\} \cup\{(N, N, \ldots, N)\}
$$

In this situation

$$
\mathbf{V}^{(N, \ldots, N)}=H^{N, p}\left(I_{1}\right) \otimes_{a} \cdots \otimes_{a} H^{N, p}\left(I_{d}\right) .
$$

The norm in $\mathbf{V}^{\mathbf{n}}$ is also given by (5.11). In particular, the norm

$$
\|f\|_{\text {mix }}:=\|f\|_{(N, \ldots, N)}=\left(\sum_{\mathbf{k} \leq(N, \ldots, N)} \int_{\mathbf{I}}\left|\partial^{\mathbf{k}} f\right|^{p} \mathrm{~d} x\right)^{1 / p}
$$


in $\mathbf{V}^{(N, \ldots, N)}$ is a crossnorm. Since in $\mathbf{V}_{\|\cdot\|_{\mathcal{N}}}$ we take

$$
\|f\|_{\mathcal{N}}:=\left(\sum_{\mathbf{n} \in \mathcal{N}}\|f\|_{\mathbf{n}}^{p}\right)^{1 / p}
$$

which is equivalent to the $\|\cdot\|_{\mathbf{m i x}^{-}}{ }^{-n o r m}$, by Corollary 5.10, we obtain

$$
\begin{aligned}
\|\cdot\|_{\text {mix }} \bigotimes_{j=1}^{d} H^{N, p}\left(I_{j}\right) & =\bigcap_{\mathbf{n} \in \mathcal{N}} \overline{H^{n_{1}, p}\left(I_{1}\right) \otimes_{a} \cdots \otimes_{a} H^{n_{d}, p}\left(I_{d}\right)}\|\cdot\|_{\mathbf{n}} \\
& \varsubsetneqq \bigcap_{|\mathbf{n}| \leq N} \overline{H^{n_{1}, p}\left(I_{1}\right) \otimes_{a} \cdots \otimes_{a} H^{n_{d}, p}\left(I_{d}\right)}\|\cdot\|_{\mathbf{n}}
\end{aligned}
$$

Thus,

$$
\|\cdot\|_{\text {mix }} \bigotimes_{j=1}^{d} H^{N, p}\left(I_{j}\right) \varsubsetneqq\|\cdot\|_{N, p} \bigotimes_{j=1}^{d} H^{N, p}\left(I_{j}\right) .
$$

In particular, for $p=2$, we have

$$
\|\cdot\|_{\text {mix }} \bigotimes_{j=1}^{d} H^{N}\left(I_{j}\right) \varsubsetneqq\|\cdot\|_{N, 2} \bigotimes_{j=1}^{d} H^{N}\left(I_{j}\right)=H^{N}(\mathbf{I}) .
$$

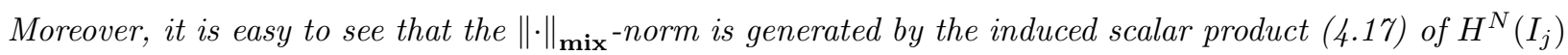
for $1 \leq j \leq d$ and satisfies condition (4.6). This fact implies that Theorem 5.1 holds for the Hilbert tensor space $\|\cdot\|_{\text {mix }} \bigotimes_{j=1}^{d} H^{N}\left(I_{j}\right)$.

Thus, a natural question arising in the above example is, whether Theorem 5.1 holds for the Hilbert tensor space $H^{N}(\mathbf{I})$ characterised by (5.13).

From Proposition 5.9, there are different equivalent versions how to define the minimal subspace $U_{j, \min }(\mathbf{v})=\operatorname{span}\left\{v_{j}^{(i)}: 1 \leq i \leq r\right\}$ for $\mathbf{v}=\sum_{i=1}^{r} \bigotimes_{j=1}^{d} v_{j}^{(i)}$. Here, we can state the following.

Corollary 5.13 Let $\mathcal{N} \subset \mathbb{N}_{0}^{d}$ be an admissible index set. For each $\mathbf{v} \in \mathbf{V}^{\left(N_{1}, \ldots, N_{d}\right)}$,

$$
U_{j, \min }(\mathbf{v})=\left\{\left(i d_{j} \otimes \varphi_{[j]}\right)(\mathbf{v}): \varphi_{[j]} \in\left({ }_{a} \bigotimes_{k \neq j} V_{k}^{\left(n_{k}\right)}\right)^{\prime}\right\} \subset V_{j}^{\left(N_{j}\right)}
$$

holds for $1 \leq j \leq d$.

Corollary 5.13 cannot be extended as Corollary 4.9 for the Banach space case. A simple counter-example is $f \in C^{1}(I \times J)$ with $f \notin C^{1}$. Choose $\varphi \in C^{1}(J)^{*}$ as $\varphi=\delta_{\eta}^{\prime}$. Then $\varphi(f)(x)=-f^{\prime}(x+\eta) \in C^{0}(I)$, but $\varphi(f)$ is not in $C^{1}(I)$ in contrast to Corollary 5.13. While, in Corollary 5.13, we could take functionals from $\left({ }_{a} \bigotimes_{k \neq j} V_{k}^{\left(n_{k}\right)}\right)^{\prime}$ for any $\mathbf{n}$ bounded by $n_{k} \leq N_{k}$, we now have to restrict the functionals to $\mathbf{n}=\mathbf{0}$. Because of the notation $V_{k}^{(0)}=V_{k}$, the definition coincides with the usual one:

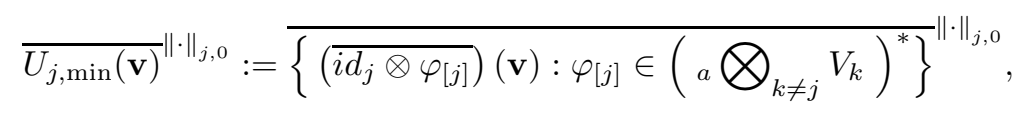

where the completion is performed with respect to the norm $\|\cdot\|_{j, 0}$ of $V_{j}^{(0)}$.

In the following we show that the same results can be derived as in the standard case. Condition (4.6) used before has to be adapted to the situation of the intersection space. Consider the tuples $\mathbf{N}_{j}=\left(0, \ldots, 0, N_{j}, 0, \ldots, 0\right) \in \mathcal{N}$ from $(5.5 \mathrm{c})$ and the corresponding tensor space

$$
\mathbf{V}^{\left(\mathbf{N}_{j}\right)}=V_{1} \otimes_{a} \ldots \otimes_{a} V_{j-1} \otimes_{a} V_{j}^{\left(N_{j}\right)} \otimes_{a} V_{j+1} \otimes \ldots \otimes_{a} V_{d}
$$

endowed with the norm $\|\cdot\|_{\mathbf{N}_{j}}$. From now on, we denote by $\|\cdot\|_{\vee\left(\mathbf{N}_{j}\right)}$ the injective norm defined from the Banach spaces $V_{1}, \ldots, V_{j-1}, V_{j}^{\left(N_{j}\right)}, V_{j+1}, \ldots, V_{d}$. 
Theorem 5.14 Assume that $\mathbf{V}_{\|\cdot\|_{\mathcal{N}}}$ is a reflexive Banach space induced by the intersection of the set of Banach tensor spaces $\left\{\mathbf{V}_{\|\cdot\|_{\mathbf{n}}}^{(\mathbf{n})}: \mathbf{n} \in \mathcal{N}\right\}$ and

$$
\|\cdot\|_{\vee\left(\mathbf{N}_{j}\right)} \lesssim\|\cdot\|_{\mathbf{N}_{j}} \quad \text { for all } 1 \leq j \leq d
$$

Then, for each $\mathbf{v} \in \mathbf{V}_{\|\cdot\|_{\mathcal{N}}}$, there exists $\mathbf{w} \in \mathcal{T}_{\mathbf{r}}$ such that

$$
\|\mathbf{v}-\mathbf{w}\|=\min _{\mathbf{u} \in \mathcal{T}_{\mathbf{r}}}\|\mathbf{v}-\mathbf{u}\|
$$

Lemma 5.15 Assume that $\mathbf{V}_{\|\cdot\|_{\mathcal{N}}}$ is a Banach space induced by the intersection of the set of Banach tensor spaces $\left\{\mathbf{V}_{\|\cdot\|_{\mathbf{n}}}^{(\mathbf{n})}: \mathbf{n} \in \mathcal{N}\right\}$ satisfying assumption (5.16). Let $\boldsymbol{\varphi}_{[j]} \in{ }_{a} \bigotimes_{k \neq j} V_{k}^{*}$ and $\mathbf{v}_{n}, \mathbf{v} \in \mathbf{V}_{\|\cdot\|_{\mathcal{N}}}$ with $\mathbf{v}_{n} \rightarrow \mathbf{v}$. Then weak convergence $\left(\overline{i d_{j} \otimes \varphi_{[j]}}\right)\left(\mathbf{v}_{n}\right) \rightarrow\left(\overline{i d_{j} \otimes \varphi_{[j]}}\right)(\mathbf{v})$ holds in $V_{j}^{\left(N_{j}\right)}$.

Proof. Repeat the proof of Lemma 4.12 and note that $\varphi_{j} \in\left(V_{j}^{\left(N_{j}\right)}\right)^{*}$ composed with an elementary tensor $\varphi_{[j]}=\bigotimes_{k \neq j} \varphi_{k}\left(\varphi_{k} \in V_{k}^{*}\right)$ yields $\varphi=\bigotimes_{k=1}^{d} \varphi_{k} \in{ }_{a} \bigotimes_{k=1}^{d}\left(V_{k}^{\left(n_{k}\right)}\right)^{*}$ with $n_{k}=0$ for $k \neq j$ and $n_{j}=N_{j}$. By (5.16) and Proposition 4.7b, $\varphi$ belongs to $\left(\mathbf{V}^{\left(\mathbf{N}_{j}\right)}\right)^{*}$.

Corollary 5.16 Under the assumptions of Lemma 5.15, $\overline{U_{j, \min }(\mathbf{v})}{ }^{\|\cdot\|_{j, 0}} \subset V_{j}^{\left(N_{j}\right)}$ holds for all $\mathbf{v} \in \mathbf{V}_{\|\cdot\|_{\mathcal{N}}}$ and $1 \leq j \leq d$.

Proof. Let $\mathbf{v}_{m} \in \mathbf{V}^{\left(N_{1}, \ldots, N_{d}\right)}$ be a sequence with $\mathbf{v}_{m} \rightarrow \mathbf{v} \in \mathbf{V}_{\|\cdot\|_{\mathcal{N}}}$ By definition (5.9) of the intersection norm, $\left\|\mathbf{v}_{m}-\mathbf{v}\right\|_{\mathbf{N}_{j}} \rightarrow 0$ holds for all $j$. Then (4.7) shows that $\left\|\left(\overline{i d_{j} \otimes \boldsymbol{\varphi}_{[j]}}\right)\left(\mathbf{v}-\mathbf{v}_{m}\right)\right\|_{j, N_{j}} \rightarrow 0$. Since $\left(\overline{i d_{j} \otimes \boldsymbol{\varphi}_{[j]}}\right)\left(\mathbf{v}_{m}\right) \in V_{j}^{\left(N_{j}\right)}$ by Proposition 5.9, also the limit of $\left(\overline{i d_{j} \otimes \boldsymbol{\varphi}_{[j]}}\right)(\mathbf{v})$ belongs to $V_{j}^{\left(N_{j}\right)}$.

Lemma 5.17 Assume that $\mathbf{V}_{\|\cdot\|_{\mathcal{N}}}$ is a Banach space induced by the intersection of the set of Banach tensor spaces $\left\{\mathbf{V}_{\|\cdot\|_{\mathbf{n}}}^{(\mathbf{n})}: \mathbf{n} \in \mathcal{N}\right\}$ satisfying assumption (5.16). For $\mathbf{v}_{m} \in \mathbf{V}^{\left(N_{1}, \ldots, N_{d}\right)}$ assume $\mathbf{v}_{m} \rightarrow \mathbf{v} \in \mathbf{V}_{\|\cdot\|_{\mathcal{N}}}$. Then

$$
\operatorname{dim} U_{j, \min }(\mathbf{v})=\operatorname{dim} \overline{U_{j, \min }(\mathbf{v})}\|\cdot\|_{j, 0} \leq \liminf _{m \rightarrow \infty} \operatorname{dim} U_{j, \min }\left(\mathbf{v}_{m}\right) \quad \text { for all } 1 \leq j \leq d .
$$

Proof. We can repeat the proof from Theorem 4.13.

Finally, in a similar way as Proposition 5.2, we can also obtain the following statement.

Proposition 5.18 Assume that $\mathbf{V}_{\|\cdot\|_{\mathcal{N}}}$ is a Banach space induced by the intersection of the set of Banach tensor spaces $\left\{\mathbf{V}_{\|\cdot\|}^{(\mathbf{n})}: \mathbf{n} \in \mathcal{N}\right\}$ satisfying the assumption (5.16). Then the set $\mathcal{T}_{\mathbf{r}}$ is weakly closed.

Proof of Theorem 5.14. Is a consequence of Theorem 2.8 and Proposition 5.18.

Example 5.19 Now, we return to $H^{N}(\mathbf{I})$ characterised as an intersection of Hilbert tensor spaces by (5.13). Recall that $\|\cdot\|_{\mathbf{N}_{j}}$ is a reasonable crossnorms in

$$
\mathbf{V}^{\left(\mathbf{N}_{j}\right)}=L^{2}\left(I_{1}\right) \otimes_{a} \cdots \otimes_{a} L^{2}\left(I_{j-1}\right) \otimes_{a} H^{N, 2}\left(I_{j}\right) \otimes_{a} L^{2}\left(I_{j+1}\right) \otimes \cdots \otimes_{a} L^{2}\left(I_{d}\right)
$$

for $1 \leq j \leq d$. Then condition (5.16) holds and we obtain the existence of a best $\mathcal{T}_{\mathbf{r}}$ approximation in this spaces.

For Hilbert spaces, Uschmajew [23] has proved the existence of minimisers of (1.3) using particular properties of Hilbert spaces. 


\subsection{Some consequences of the best $\mathcal{T}_{\mathrm{r}}$ approximation in a Hilbert tensor space}

In this section we assume that the Hilbert space $\mathbf{V}_{\|\cdot\|}:={ }_{\|\cdot\|} \bigotimes_{j=1}^{d} V_{j}$, which is the completion of $\mathbf{V}:=$ ${ }_{a} \bigotimes_{j=1}^{d} V_{j}$ with respect to $\|\cdot\|$, has the property that the set $\mathcal{T}_{\mathbf{r}}$ is weakly closed. Then for each $\mathbf{r} \in \mathbb{N}_{0}^{d}$ with $\mathbf{r} \geq \mathbf{1}$, we are to be able to define a map from $\mathbf{V}_{\|\cdot\|}$ to $[0, \infty)$, by means of

$$
\|\mathbf{u}\|_{\mathbf{r}}:=\max \left\{|\langle\mathbf{v}, \mathbf{u}\rangle|: \mathbf{v} \in \mathcal{T}_{\mathbf{r}},\|\mathbf{v}\|=1\right\} .
$$

Observe, that if $\|\cdot\|$ is induced by the scalar products of $V_{j}$, then $\|\cdot\|_{1}=\|\cdot\|_{\vee}$. Now, we obtain the following result.

Theorem 5.20 Assume that in the Hilbert space $\mathbf{V}_{\|\cdot\|}$ the set $\mathcal{T}_{\mathbf{r}}$ is weakly closed. Then for each $\mathbf{r} \in \mathbb{N}_{0}^{d}$ with $\mathbf{r} \geq \mathbf{1}$, the following statements hold.

(a) For each $\mathbf{u} \in \mathbf{V}_{\|\cdot\|}$ the equality

$$
\|\mathbf{u}\|_{\mathbf{r}}^{2}=\|\mathbf{u}\|^{2}-\min _{\mathbf{v} \in \mathcal{T}_{\mathbf{r}}}\|\mathbf{u}-\mathbf{v}\|^{2}
$$

holds.

(b) If $\mathbf{s} \in \mathbb{N}_{0}^{d}$ satisfies $\mathbf{r} \leq \mathbf{s}$, then $\|\mathbf{u}\|_{\mathbf{r}} \leq\|\mathbf{u}\|_{\mathbf{s}}$ for all $\mathbf{u} \in \mathbf{V}_{\|\cdot\| \cdot}$.

(c) $\|\cdot\|_{\mathbf{r}}$ is a norm on $\mathbf{V}_{\|\cdot\|}$.

Proof. To prove (a) let $\mathcal{D}=\left\{\mathbf{w}: \mathbf{w} \in \mathcal{T}_{\mathbf{r}}\right.$ and $\left.\|\mathbf{w}\|=1\right\}$. Then

$$
\min _{\mathbf{v} \in \mathcal{T}_{\mathbf{r}}}\|\mathbf{u}-\mathbf{v}\|=\min _{\mathbf{w} \in \mathcal{D}, \lambda \in \mathbb{R}}\|\mathbf{u}-\lambda \mathbf{w}\| \text {. }
$$

Note that $\|\mathbf{u}-\lambda \mathbf{w}\|^{2}=\|\mathbf{u}\|^{2}-2 \lambda\langle\mathbf{u}, \mathbf{w}\rangle+\lambda^{2}$. The minimum of $\|\mathbf{u}-\lambda \mathbf{w}\|_{2}^{2}$ for $\mathbf{w} \in \mathcal{D}$ is obtained for $\lambda=\langle\mathbf{u}, \mathbf{w}\rangle>0$ and equals $\|\mathbf{u}-\lambda \mathbf{w}\|_{2}^{2}=\|\mathbf{u}\|_{2}^{2}-|\langle\mathbf{u}, \mathbf{w}\rangle|^{2}$. Thus,

$$
\min _{\mathbf{v} \in \mathcal{T}_{\mathbf{r}}}\|\mathbf{u}-\mathbf{v}\|^{2}=\min _{\lambda \in \mathbb{R}, \mathbf{w} \in \mathcal{D}}\|\mathbf{u}-\lambda \mathbf{w}\|^{2}=\min _{\mathbf{w} \in \mathcal{D}}\|\mathbf{u}-\langle\mathbf{u}, \mathbf{w}\rangle \mathbf{w}\|^{2}=\|\mathbf{u}\|^{2}-\max _{\mathbf{w} \in \mathcal{D}}|\langle\mathbf{u}, \mathbf{w}\rangle|^{2}
$$

and from (5.17) statement (a) follows.

Since $\mathbf{r} \leq \mathbf{s}$ implies $\mathcal{T}_{\mathbf{r}} \subset \mathcal{T}_{\mathbf{s}}$, statement (b) follows from (5.17).

To prove (c) note that the norm axiom $\|\lambda \mathbf{u}\|_{\mathbf{r}}=|\lambda|\|\mathbf{u}\|_{\mathbf{r}}$ and the triangle inequality are standard. To prove that $\mathbf{u} \neq \mathbf{0}$ implies $\|\mathbf{u}\|_{\mathbf{r}}>0$, note that if $\|\mathbf{u}\|_{\mathbf{r}}=0$ we have $\langle\mathbf{u}, \mathbf{v}\rangle=0$ for all $\mathbf{v} \in \mathcal{T}_{\mathbf{r}}$. Since $\operatorname{span} \mathcal{T}_{\mathbf{r}}$ is dense in $\mathbf{V}_{\|\cdot\|}$, we obtain that $\mathbf{u}=\mathbf{0}$.

Let $V_{1}=\mathbb{R}^{n_{1}}$ and $V_{2}=\mathbb{R}^{n_{2}}$ be equipped with the usual Euclidean norm. Then $V_{1} \otimes_{a} V_{2}$ is isomorphic to matrices from $\mathbb{R}^{n_{1} \times n_{2}}$ with the Frobenius norm $\|\cdot\|$. It is not difficult to see that $\|\mathbf{u}\|_{(1,1)}$ coincides with $\sigma_{1}$, the first singular value of the singular value decomposition of $\mathbf{u}$.

\section{References}

[1] A. Ammar, B. Mokdad, F. Chinesta, and R. Keunings: A new family of solvers for some classes of multidimensional partial differential equations encountered in kinetic theory modelling of complex fluids. Journal of Non-Newtonian Fluid Mechanics, 1393 (2006) 153-176.

[2] C.J. Appellof and E.R. Davidson: Strategies for analyzing data from video fluorometric monitoring of liquid-chromatographic effluents. Analytical Chemistry, 5313 (1981) 2053-2056.

[3] G. Berkooz, P. Holmes, and J. L. Lumley: The proper orthogonal decomposition in the analysis of turbulent flows. Annual review of fluid mechanics, 25 (1993) 539-575.

[4] E. Cances, V. Ehrlacher, and T. Lelievre: Convergence of a greedy algorithm for high-dimensional convex nonlinear problems. In arXiv arXiv(1004.0095v1 [math.FA]), April 2010.

[5] J. D. Carroll and J. J. Chang: Analysis of individual differences in multidimensional scaling via an n-way generalization of Eckart-Young decomposition. Psychometrika,35 (1970) 283-319. 
[6] V. de Silva and L.-H. Lim: Tensor rank and ill-posedness of the best low-rank approximation problem. SIAM Journal of Matrix Analysis Appl. 30 (2008), 1084-1127.

[7] R.A. DeVore and G.G. Lorentz: Constructive approximation. Springer-Verlag, Berlin, 1993.

[8] A. Doostan and G. Iaccarino: A least-squares approximation of partial differential equations with highdimensional random inputs. J. Comput. Phys. 228 (12) (2009) 4332-4345.

[9] A. Falcó: Algorithms and numerical methods for high dimensional financial market models. Revista de Economia Financiera, 2010 (In press).

[10] W. H. Greub: Linear Algebra. Graduate Text in Mathematics, Springer-Verlag, fourth edition, 1981.

[11] A. Grothendieck: Résumé de la théorie métrique des produit tensoriels topologiques. Bol. Soc. Mat. São Paulo 8 (1953/56) 1-79.

[12] W. Hackbusch and S. Kühn: A new scheme for the tensor representation. J. Fourier Anal. Appl. 15 (2009) 706-722.

[13] F. L. Hitchcock: The expression of a tensor or a polyadic as a sum of products. Journal of Mathematics and Physics 6 (1927) 164-189.

[14] T. G. Kolda and B. W. Bader: Tensor decompositions and applications. SIAM Review, 51 (2009) 455-500.

[15] L. De Lathauwer and J. Vandewalle: Dimensionality reduction in higher-order signal processing and rank- $\left(r_{1}, r_{2}, \ldots, r_{n}\right)$ reduction in multilinear algebra. Linear Algebra Appl., 391 (2004) 31-55.

[16] W. A. Light and E. W. Cheney: Approximation theory in tensor product spaces. Lecture Notes in Mathematics 1169, Springer-Verlag, 1985.

[17] R. Meise and D. Vogt: Introduction to functional analysis. Clarendon Press, Oxford, 1997.

[18] A. Nouy: A generalized spectral decomposition technique to solve a class of linear stochastic partial differential equations. Computer Methods in Applied Mechanics and Engineering, 96 (45-48) (2007) 4521-4537.

[19] A. Nouy: Proper generalized decompositions and separated representations for the numerical solution of high dimensional stochastic problems. Archives of Computational Methods in Engineering, 2010, In press.

[20] I.V. Oseledets and E.E. Tyrtyshnikov: TT-cross approximation for multidimensional arrays. Linear Algebra Appl. 432 (2010) 70-88.

[21] B. Simon: Uniform crossnorms. Pacific J. Math. 46 (1973) 555-560.

[22] L. R. Tucker: Some mathematical notes on three-mode factor analysis. Psychometrika, 31 (1966) 279311.

[23] A. Uschmajew: Convex maximization problems on non-compact Stiefel manifolds with application to orthogonal tensor approximations. Numer. Math, 115 (2010) 309-331.

[24] M. A. O. Vasilescu and D. Terzopoulos: Multilinear analysis of image ensembles: tensorfaces. ECCV 2002:Proceedings of the 7th European Conference on Computer Vision, Lecture Notes in Comput. Sci. 2350, 447-460. Springer, 2002.

[25] G. Vidal: Efficient classical simulation of slightly entangled quantum computations. Phys. Rev. Letters 91 (2003), 147902.

[26] H. Wang and N. Ahuja: Compact representation of multidimensional data using tensor rank-one decomposition. ICPR 2004:Proceedings of the 17th International Conference on Pattern Recognition, volume 1, (2004) 44-47 